\title{
ON THE FUNCTIONAL EQUATIONS INVOLVING NONLINEAR GENERALIZED P-COMPACT OPERATORS
}

\author{
BY \\ W. V. PETRYSHYN( $\left.{ }^{1}\right)$ AND T. S. TUCKER $\left({ }^{2}\right)$
}

Introduction. In his recent papers [23], [24], [25] Petryshyn has introduced and studied a class of projectionally compact ( $P$-compact) nonlinear mappings of a real Banach space with property $(\pi)_{K}$ into itself. The main result of this study was an elementary and essentially a constructive proof of two fixed point theorems for bounded [23], [24] and unbounded [25] $P$-compact operators which were then used to derive a number of results concerning the existence and construction of solutions of various classes of nonlinear equations. Since, as was shown in [25], $P$-compact operators include, among others, completely continuous, quasicompact, and various classes of monotone operators (in a Hilbert space), the fixed point theorems (for balls about the origin) of Schauder [34], Rothe [32], Krasnoselsky [17], Altman [1] and Kaniel [14] as well as certain existence theorems for monotone operators of Zarantonello [41], Kacurowski [13], Minty [21], Browder [2], [3], Dolph and Minty [10] and others were deduced from the results on $P$-compact operators. Further results for $P$-compact operators or their extensions have been recently obtained by Lees and Schultz [18], De Figueiredo [8], [9] and Tucker [36].

The purpose of this paper is to continue the study and to extend the results for $P$-compact operators obtained in [23], [24], [25] to generalized $P$-compact or $P_{\gamma}$-compact operators introduced in [28]. One of our objectives is to show that many properties and results which are true for completely continuous operators carry over to generalized $P$-compact operators.

We now outline briefly the main results of this paper.

In $\$ 1$ we introduce and discuss some basic definitions to be used in this paper.

In $\$ 2$ we discuss further properties of $P$-compact and generalized $P$-compact operators. The main result of this section is Theorem 2.4 which asserts that under certain conditions "the Fréchet derivative $A^{\prime}(x)$ is $P$-compact if and only if $A(x)$ is $P$-compact."

In $\S 3$ we discuss certain properties of linear $P$-compact operators. The main result of this section is the characterization Theorem 3.3 which asserts that "a symmetric linear mapping $A$ of a Hilbert space into itself is $P$-compact if and only if $A=S+T$ where $S \leqq 0$ and $T$ is completely continuous."

Received by the editors August 31, 1967.

(1) Present address: Department of Mathematics, Rutgers University, New Brunswick, New Jersey.

(2) Present address: Recourse Management Corporation, Bethesda, Maryland. 
In $\S 4$ the fixed point theorems of [23], [24], [25] are extended to generalized $P$ compact operators to cover fixed point theorems for bounded closed convex sets with an interior. The main result of this section is Theorem 4.4 for unbounded $P_{\gamma}$-compact operators from which we then derive a number of known fixed point theorems mentioned above for convex sets as well as some recent results of Yamamuro [40], Hanani, Netanyahu af̂́d Reichaw-Reichbach [12] and others.

In $\$ 5$ we apply our general fixed point theorem to the solution of various classes of nonlinear equations. In particular, we deduce the results of Krasnoselsky [16], [17], Ehrmann [11], certain results related to fixed point theorems of Browder [4] and others.

In $\S 6$ we first prove the convergence of an iteration method for the construction of fixed points of nonexpansive $P_{1}$-compact mappings by utilizing the results and arguments of Browder-Petryshyn [6] and Krasnoselsky [16]; then we study a general (nonprojective) approximation method in the solution of functional equations. As special cases we deduce the corresponding results of Petryshyn [24] and Vainikko [39].

1. Basic definitions. In this paper it will always be assumed that $X$ is a real Banach space such that there exists a pair of sequences $\left(\left\{X_{n}\right\},\left\{P_{n}\right\}\right)$, where each $X_{n}$ is a finite dimensional subspace of $X$ and $P_{n}$ is a linear projection of $X$ onto $X_{n}$ such that $P_{n} x \rightarrow x$ as $n \rightarrow \infty$ for each $x$ in $X$. The system $\left(\left\{X_{n}\right\},\left\{P_{n}\right\}\right)$ having the above property is said to be projectionally complete [27], [30]. In what follows we use " $\rightarrow$ " and " $\rightarrow$ " to denote strong and weak convergence in $X$, respectively, and $H$ to denote a real Hilbert space.

Since some of the terms used in this paper are not universally accepted by people working in the field, to avoid possible confusion, we first state the following definitions. Let $A$ be a (not necessarily linear) mapping of a (not necessarily linear) set $D(A) \subset X$ into $X . A$ is said to be precompact if $A$ maps every bounded set in $D(A)$ into a precompact set in $X ; A$ is completely continuous if $A$ is continuous and precompact; $A$ is strongly continuous if $\left\{x_{n}\right\} \subset D(A), x \in D(A)$, and $x_{n} \rightarrow x$ implies $A x_{n} \rightarrow A x ; A$ is weakly continuous if $\left\{x_{n}\right\} \subset D(A), x \in D(A)$, and $x_{n} \rightarrow x$ implies $A x_{n} \rightarrow A x ; A$ is hemicontinuous if $A$ is continuous from line segments in $D(A)$ to the weak topology in $X ; A$ is demiclosed if $\left\{x_{n}\right\} \subset D(A), x_{n} \rightarrow x, A x_{n} \rightarrow y$ imply $x \in D(A)$ and $A x=y ; A$ is closed if $\left\{x_{n}\right\} \subset D(A), x_{n} \rightarrow x, A x_{n} \rightarrow y$ imply $x \in D(A)$ and $A x=y ; A$ is strongly closed if $\left\{x_{n}\right\} \subset D(A), x_{n} \rightarrow x, A x_{n} \rightarrow y$ imply $x \in D(A)$ and $A x=y ; A$ is bounded if $A$ maps bounded sets in $D(A)$ into bounded sets in $X$; $A$ is monotone increasing on $D(A)=X=H$ if $(A x-A y, x-y) \geqq 0$ for all $x$ and $y$ in $H ; A$ is monotone decreasing if $-A$ is monotone increasing. We also use $B_{r}\left(x_{0}\right)$ and $S_{r}\left(x_{0}\right)=\partial B_{r}\left(x_{0}\right)$ to denote the ball (i.e., $B_{r}\left(x_{0}\right)=\left\{x \in X \mid\left\|x-x_{0}\right\| \leqq r\right\}$ ) and its boundary.

The class of operators to be studied in this paper is described by the following definition. 
Definition 1.1 [28]. A nonlinear operator $A$ of $D(A)(\subset X)$ into $X$ is called a generalized $P$-compact mapping or $P_{\gamma}$-compact if $P_{n} A$ is continuous in $X_{n}$ for all sufficiently large $n$ and if there exists a constant $\gamma \geqq 0$ such that for any $p$ dominating $\gamma$ (i.e., $p \geqq \gamma$ if $\gamma>0$ and $p>\gamma$ if $\gamma=0$ ) and any bounded sequence $\left\{x_{n}\right\}$ with $x_{n} \in$ $X_{n} \cap D(A)$ the strong convergence of the sequence $\left\{P_{n} A x_{n}-p x_{n}\right\}$ implies the existence of a strongly convergent subsequence $\left\{x_{n_{i}}\right\}$ and an element $x \in D(A)$ such that $x_{n_{i}} \rightarrow x$ and $P_{n_{i}} A x_{n_{i}} \rightarrow A x$ as $n_{i} \rightarrow \infty$.

REMARK 1.1. It was pointed out in [28] that the concept of $P_{\gamma}$-compactness is a generalization of the concept of projectional compactness or P-compactness introduced and studied in [23], [24], [25]. Indeed, when in Definition 1.1 we take $\gamma=0, D(A)=X$ and $\left(\left\{X_{n}\right\},\left\{P_{n}\right\}\right)$ so that

$(\pi)_{K} P_{n} X=X_{n} ; X_{n} \subset X_{n+1}(n=1,2, \ldots) ; \bigcup_{n=1}^{\infty} X_{n}=X ;\left\|P_{n}\right\| \leqq K \quad(K \geqq 1)$,

then it is easy to see that condition $(\pi)_{K}$ implies the projectional completeness of $\left(\left\{X_{n}\right\},\left\{P_{n}\right\}\right)$. Thus in this case, the concept of $P_{0}$-compactness, according to Definition 1.1 , is just the concept of $P$-compactness. In what follows we shall refer to $P_{0}$-compactness as $P$-compactness although we no longer require that $\left(\left\{X_{n}\right\},\left\{P_{n}\right\}\right)$ satisfy condition $(\pi)_{K}$ or that $D(A)$ be the entire space $X$. We remark that upon examination of the proofs of the fixed point theorems in [23], [24], [25] it is clear that the only properties of $A$ and $X$ which were used are contained in the requirement that $A$ be $P_{1}$-compact according to Definition 1.1 as a mapping from $B_{r}(0)$ to $X$. It turns out that the class of operators defined by the generalized Definition 1.1 is not only larger but, at the same time, is more suitable for theoretical discussions and applicational purposes.

2. Properties and examples of nonlinear $P_{y}$-compact operators. It was shown in [24] that the class of bounded $P$-compact operators $A$ with $D(A)=X$ contains as its subclasses all closed precompact operators and, in particular, completely continuous operators (and strongly continuous operators if $X$ is reflexive); all operators $A$ such that $-A$ is quasicompact (see [14], [24]); and all bounded continuous, demicontinuous and weakly continuous monotone decreasing operators $A$ defined on $H$. Consequently, the various fixed point theorems in [34], [32], [17], [1], [14] and existence theorems in [21], [2], [3] for monotone operators were deduced as special cases of the fixed point theorems for $P$-compact operators.

In this section we discuss further classes and properties of nonlinear (not necessarily bounded) $P$-compact operators. At the same time we obtain some new results for monotone operators in $H$.

THEOREM 2.1. If $A$ is a hemicontinuous monotone decreasing operator of $D(A)=H$ into $H$ such that $P_{n} A P_{n} x \rightarrow A x(n \rightarrow \infty)$ for each $x$ in $H$, then $A$ is $P$-compact.

Proof. First note that since $P_{n} A$, as a mapping of the finite-dimensional space $X_{n}$ into $X_{n}$, is hemicontinuous and monotone decreasing, it is also continuous [15]. 
Now let $\left\{x_{n}\right\}$ be any bounded sequence in $X$ with $x_{n} \in X_{n}$ so that for some $p>0$

$$
g_{n} \equiv P_{n} A x_{n}-p x_{n} \rightarrow g \text { in } X \text { as } n \rightarrow \infty .
$$

Since $-A$ is monotone increasing and $p>0$, the operator $T=-A+p I$ is strongly monotone on $H$, i.e.,

$$
p\|x-y\|^{2} \leqq(T x-T y, x-y) \text { for all } x, y \in H,
$$

and $P_{n} T x_{n} \rightarrow-g$. Since $\left\{x_{n}\right\}$ is bounded, there exists a subsequence $\left\{x_{m}\right\}$ of $\left\{x_{n}\right\}$ and an element $x_{0}$ in $X$ such that $x_{m} \rightarrow x_{0}$. Since $P_{m} x_{m}=x_{m}$,

$$
\begin{aligned}
p\left\|x_{m}-P_{m} x_{0}\right\| & \leqq\left(T x_{m}-T P_{m} x_{0}, x_{m}-P_{m} x_{0}\right)=\left(P_{m} T x_{m}-P_{m} T P_{m} x_{0}, x_{m}-P_{m} x_{0}\right) \\
& =\left(P_{m} T x_{m}, x_{m}\right)-\left(P_{m} T P_{m} x_{0}, x_{m}\right)-\left(P_{m} T x_{m}, P_{m} x_{0}\right)+\left(P_{m} T P_{m} x_{0}, P_{m} x_{0}\right) .
\end{aligned}
$$

By assumption, $P_{m} T x_{m} \rightarrow-g, x_{m} \rightarrow x_{0}$ and $P_{m} A P_{m} x_{0} \rightarrow A x_{0}$, so passing to the limit in the last inequality, we see that $\left\|x_{m}-P_{m} x_{0}\right\| \rightarrow 0$ as $m \rightarrow \infty$. This and the inequality

$$
\left\|x_{m}-x_{0}\right\| \leqq\left\|x_{m}-P_{m} x_{0}\right\|+\left\|P_{m} x_{0}-x_{0}\right\|
$$

imply that $x_{m} \rightarrow x_{0}$ as $m \rightarrow \infty$.

To show that $P_{m} A x_{m} \rightarrow A x_{0}$, we note first that for $\tilde{A}=-A, P_{m} \tilde{A} x_{m}=-g_{m}-p x_{m}$ $\rightarrow-g-p x_{0}$ as $n \rightarrow \infty$, and that for any $y$ in $H$,

$$
\left(P_{m} \tilde{A} P_{m} y-P_{m} \tilde{A} x_{m}, P_{m} y-x_{m}\right)=\left(\tilde{A} P_{m} y-\tilde{A} x_{m}, P_{m} y-x_{m}\right) \geqq 0 .
$$

Thus on passage to the limit, we derive the inequality

$$
\left(\tilde{A} y+g+p x_{0}, y-x_{0}\right) \geqq 0 \text { for all } y \text { in } H .
$$

If in the last inequality we take $y=x_{0}+t z$ for any $z$ in $H$ and $t>0$, then it reduces to

$$
\left(\tilde{A}\left(x_{0}+t z\right)+g+p x_{0}, t z\right) \geqq 0
$$

or, after cancelling the positive factor $t>0$, to

$$
\left(\tilde{A}\left(x_{0}+t z\right)+g+p x_{0}, z\right) \geqq 0 .
$$

Since $A$ (and hence $\tilde{A}$ ) is hemicontinuous and $x_{0}+t z \rightarrow x_{0}$ as $t \rightarrow 0^{+}$, we obtain the relation

$$
\left(\tilde{A} x_{0}+g+p x_{0}, z\right) \geqq 0 \text { for all } z \text { in } H .
$$

Similarly, taking $y=x_{0}-t z$ with $t>0$, we get

$$
\left(\tilde{A} x_{0}+g+p x_{0}, z\right) \leqq 0 \text { for all } z \text { in } H .
$$

The two inequalities imply that $A x_{0}=g+p x_{0}$, so $A$ is $P$-compact.

COROllary 2.1. Every continuous monotone decreasing mapping $A$ of $H$ into $H$ is $P$-compact. 
Proof. The assertion follows immediately from Theorem 2.1 since if $A$ is continuous in $H$, then $A$ is certainly hemicontinuous and $P_{n} A P_{n} x \rightarrow A x$ for each $x$ in $H$.

COROllaRY 2.2. If $A$ satisfies the conditions of Theorem 2.1 (and in particular of Corollary 2.1), then for any given $\mu>0$ the mapping $A-\mu I$ is one-to-one and onto.

Proof. This is an immediate consequence of Theorem 2.1 and Corollary 2 in [25].

In our discussion below we will need the following simple lemma.

Lemma 2.1. For the class of P-compact mappings $A$ of $X$ into $X$, the following assertions are valid:

(a) The identity map $I: X \rightarrow X$ is not P-compact, but $-I$ is P-compact.

(b) If $A$ is $P$-compact then $-A$ is not necessarily $P$-compact, but $-A$ is $P$-compact with $p<0$. (See [24] for the discussion of the case $p<0$.)

(c) If $A$ is $P$-compact and $a \geqq 0$, then aA is also P-compact.

(d) If $A$ and $B$ are $P$-compact, then $A-B$ need not be P-compact.

(e) If $A$ is $P$-compact and $f$ is a fixed element in $X$, then $A+f$ and $A-f$ are $P$ compact.

(f) If $A$ is $P$-compact and $B$ is completely continuous, then the mapping $(1-t) A$ $+t B$ is $P$-compact for any fixed $t$ in $[0,1]$.

Proof. The assertions (a)-(e) are obvious while the proof of (f) is essentially given in [25] and, therefore, will be omitted.

We now derive a number of results for the Lipschitzian type mappings which we shall use in the section on fixed point theorems.

THEOREM 2.2. If $A$ is a nonlinear mapping of $D(A)=X$ into $X$ such that for some constant $L>0$

$$
\|A x-A y\| \leqq L\|x-y\| \text { for all } x \text { and } y \text { in } X,
$$

then $A$ is $P_{\gamma}$-compact with any fixed $\gamma>K L$, where $K \geqq 1$ is the uniform bound on the sequence $\left\{P_{n}\right\}$.

Proof. First, since $A$ is Lipschitzian, $P_{n} A$ is continuous in $X_{n}$ for each $n$. Next, since $P_{n} x \rightarrow x$ as $n \rightarrow \infty$ for each $x$ in $X$, the uniform boundedness principle implies that there exists a constant $K \geqq 1$ such that $\left\|P_{n}\right\| \leqq K$ for all $n$. Suppose now that $\left\{x_{n}\right\}$ is an arbitrary bounded sequence in $X$ with $x_{n} \in X_{n}$ so that for any fixed $\gamma>K L$ and any $p \geqq \gamma$,

$$
g_{n}=P_{n} A x_{n}-p x_{n} \rightarrow g \text { in } X \text { as } n \rightarrow \infty .
$$

Consider the operators $D=p I-A$ and $D_{n}=p I-P_{n} A$ and note that for each $n$ and all $x$ and $y$ in $X_{n}$

$$
\left\|D_{n} x-D_{n} y\right\| \geqq \alpha\|x-y\| \quad \text { with } \alpha=p-K L>0 .
$$


Since $p-L \geqq p-K L>0$, it follows that the mapping $B=p^{-1} A$ is a strict contraction on $X$. Hence $I-B$ is a bicontinuous mapping of $X$ onto $X$, by the usual iteration argument. Consequently, $D=p I-A$ is a one-to-one mapping of $X$ onto $X$. Thus, Theorem 2 in [29] implies that the mapping $D$ satisfies condition $(H)$ which, as was noted in [29], is precisely the condition that $A$ be $P_{\gamma}$-compact for $\gamma>K L$.

Corollary 2.3. If $A$ is a strict contraction on $X$ (i.e., $L<1$ ) and the sequence $\left\{P_{n}\right\}$ is such that $\left\|P_{n}\right\|=1$ (i.e., $K=1$ ), then $A$ is $P_{\gamma}$-compact for any fixed $\gamma>L$.

THEOREM 2.3. If $A$ is a nonexpansive mapping of $X$ into $X$ (i.e., $\|A x-A y\|$ $\leqq\|x-y\|$ for all $x$ and $y$ in $X)$, then the mapping $(A-I)$ is $P_{\gamma}$-compact with $\gamma=K-1$ if $K=1$ and with any fixed $\gamma>K-1$ if $K>1$.

Proof. It is clear that $P_{n} A$ is continuous in $X_{n}$ for each $n$. Now suppose that $\left\{x_{n}\right\}$ is any bounded sequence in $X$ with $x_{n} \in X_{n}$ so that for some $p$ dominating $\gamma$, $g_{n}=P_{n}(A-I) x_{n}-p x_{n} \rightarrow g$ as $n \rightarrow \infty$. Consider the operators $D=A-(1+p) I$ and $D_{n}=P_{n}(A-(1+p) I)$. Note that for all $x$ and $y$ in $X_{n}$ and all $n$,

$$
\left\|D_{n} x-D_{n} y\right\| \geqq \alpha\|x-y\| \quad \text { with } \alpha=1+p-K>0 .
$$

Since, under our conditions on $p, D=A-(1+p) I$ is a bicontinuous mapping of $X$ onto $X$, the conclusion of this theorem follows from Theorem 2 in [29].

COROLlaRY 2.4. If $\left\|P_{n}\right\|=1$ for each $n$, and $A$ is a nonexpansive mapping of $X$ into $X$, then $A-I$ is $P$-compact.

REMARK 2.1. Using the same arguments as in [25], one shows that if $A$ is $P_{\gamma^{-}}$ compact and $B$ is completely continuous, then $A+B$ is $P_{y}$-compact.

Let $A$ be a nonlinear mapping of $D(A)=X$ into $X$. We recall that $A x$ is said to be Fréchet-differentiable at a given point $x$ in $X$ if there exists a continuous linear operator $A^{\prime}(x)$ of $X$ into $X$ such that for any $h$ in $X$,

$$
A(x+h)-A x=A^{\prime}(x) h+w(x ; h) \quad \text { with } \frac{\|w(x ; h)\|}{\|h\|} \rightarrow 0 \quad \text { as }\|h\| \rightarrow 0,
$$

(i.e., to any given $\varepsilon>0$, there exists a $\delta=\delta(x, h)>0$ such that $\|w(x ; h)\| \leqq \varepsilon\|h\|$ if $\|h\| \leqq \delta)$. The operator $A^{\prime}(x)$ is called the Fréchet derivative at $x$. Let $X^{*}$ denote the conjugate space of $X$ with the value of the linear functional $e$ in $X^{*}$ at $x$ in $X$ denoted by $(x, e)$.

It is known [38] that if $A$ is completely continuous, then so is $A^{\prime}(x)$ at any $x$, but whether the converse is true is not known $\left({ }^{3}\right)$. If $A$ is $P$-compact, we have the following result:

$\left({ }^{3}\right)$ Added in proof. After this paper had been accepted for publication, the authors were informed by T. Tromba that R. A. Bonic, in his forthcoming paper to appear in J. Differential Geometry, has answered in negative the question posed by Vainberg [38, p. 51] by constructing examples of continuous mappings which are not completely continuous but whose derivatives are completely continuous. 
THEOREM 2.4. Suppose $X$ is reflexive and the Fréchet derivative $A^{\prime}: X \rightarrow L(X, X)\left(^{4}\right)$ is strongly continuous. Then at any given $x$ in $X$, the operator $A^{\prime}(x)$ is P-compact if and only if $A$ is $P$-compact.

Proof. Suppose that for any given $x$ in $X$ the operator $A^{\prime}(x)$ is $P$-compact. Note first that since $A^{\prime}(x)$ exists, by results in [38], $A$ is continuous, and, therefore, $P_{n} A$ is continuous in $X_{n}$ for each $n$. Suppose now that $\left\{x_{n}\right\} \subset X$ is an arbitrary bounded sequence with $x_{n} \in X_{n}$ such that for some $p>0, g_{n} \equiv P_{n} A x_{n}-p x_{n} \rightarrow g$ as $n \rightarrow \infty$. Since $\left\{x_{n}\right\}$ is bounded and $X$ is reflexive, we may assume without loss of generality that $x_{n}$ converges weakly to some element $x_{0}$ in $X$. For an arbitrary unit vector $e_{n m}$ in $X^{*}$ consider the equality

$$
\left(\left\{P_{n} A x_{n}-p x_{n}\right\}-\left\{P_{m} A x_{m}-p x_{m}\right\}, e_{n m}\right)=\left(g_{n}-g_{m}, e_{n m}\right)
$$

whence, on adding and subtracting $P_{n} A x_{0}$ and $P_{m} A x_{0}$, we get

$$
\begin{aligned}
\left(\left\{P_{n} A x_{n}-P_{n} A x_{0}-p x_{n}\right\}-\left\{P_{m} A x_{m}-P_{m} A x_{0}-p x_{m}\right\}, e_{n m}\right) \\
=\left(g_{n}-g_{m}, e_{n m}\right)+\left(\left\{P_{m} A x_{0}-P_{n} A x_{0}\right\}, e_{n m}\right) .
\end{aligned}
$$

In view of the mean-value theorem for functionals, there exist numbers $t_{n m}$ and $t_{n m}^{\prime}$ in $(0,1)$ such that the last equality reduces to

$$
\begin{aligned}
\left(\left\{P_{n} A^{\prime}\left(z_{n m}\right)\left(x_{n}-x_{0}\right)-p x_{n}\right\}-\left\{P_{m} A^{\prime}\left(z_{n m}^{\prime}\right)\left(x_{m}-x_{0}\right)-p x_{m}\right\}, e_{n m}\right) & \\
& =\left(g_{n}-g_{m}, e_{n m}\right)+\left(\left\{P_{m} A x_{0}-P_{n} A x_{0}\right\}, e_{n m}\right),
\end{aligned}
$$

where $z_{n m}=x_{0}+t_{n m}\left(x_{n}-x_{0}\right)$ and $z_{n m}^{\prime}=x_{0}+t_{n m}^{\prime}\left(x_{m}-x_{0}\right)$, from which we derive the useful equality

$$
\begin{aligned}
\left(\left\{P_{n} A^{\prime}\left(x_{0}\right) x_{n}-p x_{n}\right\}-\right. & \left.\left\{P_{m} A^{\prime}\left(x_{0}\right) x_{m}-p x_{m}\right\}, e_{n m}\right) \\
= & \left(\left\{P_{n} A^{\prime}\left(x_{0}\right)-P_{n} A^{\prime}\left(z_{n m}\right)\right\}\left(x_{n}-x_{0}\right), e_{n m}\right)+\left(g_{n}-g_{m}, e_{n m}\right) \\
& -\left(\left\{P_{m} A^{\prime}\left(x_{0}\right)-P_{m} A^{\prime}\left(z_{n m}^{\prime}\right)\right\}\left(x_{m}-x_{0}\right), e_{n m}\right)+\left(P_{m} A x_{0}-P_{n} A x_{0}, e_{n m}\right) \\
& -\left(P_{m} A^{\prime}\left(x_{0}\right) x_{0}-P_{n} A^{\prime}\left(x_{0}\right) x_{0}, e_{n m}\right) .
\end{aligned}
$$

Now by the Hahn-Banach theorem we can choose $e_{n m}$ so that $\left\|e_{n m}\right\|=1$ and

$$
\begin{aligned}
& \left(\left\{P_{n} A^{\prime}\left(x_{0}\right) x_{n}-p x_{n}\right\}-\left\{P_{m} A^{\prime}\left(x_{0}\right) x_{m}-p x_{m}\right\}, e_{n m}\right) \\
& \quad=\left\|\left\{P_{n} A^{\prime}\left(x_{0}\right) x_{n}-p x_{n}\right\}-\left\{P_{m} A^{\prime}\left(x_{0}\right) x_{m}-p x_{m}\right\}\right\| \equiv\left\|g_{n}^{\prime}-g_{m}^{\prime}\right\| .
\end{aligned}
$$

This and the preceding equality imply that

$$
\begin{aligned}
\left\|g_{n}^{\prime}-g_{m}^{\prime}\right\| \leqq & \left\|P_{n} A^{\prime}\left(x_{0}\right)-P_{n} A^{\prime}\left(z_{n m}\right)\right\|\left\|x_{n}-x_{0}\right\|+\left\|g_{n}-g_{m}\right\| \\
& +\left\|P_{m} A^{\prime}\left(x_{0}\right)-P_{m} A^{\prime}\left(z_{n m}^{\prime}\right)\right\|\left\|x_{m}-x_{0}\right\|+\left\|P_{m} A x_{0}-P_{n} A x_{0}\right\| \\
& +\left\|P_{m} A^{\prime}\left(x_{0}\right) x_{0}-P_{n} A^{\prime}\left(x_{0}\right) x_{0}\right\| .
\end{aligned}
$$

${ }^{(4)}$ We recall that $L(X, X)$ is the Banach space of all continuous linear operators of $X$ into $X$ with the topology in $L(X, X)$ being the metric topology defined by the bounds of the elements in $L(X, X)$. Then $A^{\prime}: X \rightarrow L(X, X)$ is strongly continuous means that $A^{\prime}\left(z_{n}\right) \rightarrow A^{\prime}(z)$ whenever $z_{n} \rightarrow z$ as $n \rightarrow \infty$. 
Since $\left\{t_{n m}\right\}$ and $\left\{t_{n m}^{\prime}\right\}$ belong to $(0,1)$ and $x_{n} \rightarrow x_{0}$ and $x_{m} \rightarrow x_{0}$ it is clear that $z_{n m} \rightarrow x_{0}$ independent of $m$ as $n \rightarrow \infty$ and $z_{n m}^{\prime} \rightarrow x_{0}$ independent of $n$ as $n, m \rightarrow \infty$. This, the boundedness of $\left\{x_{n}\right\}$, and the strong continuity of $A^{\prime}$ imply that the first and the third terms in the above inequality go to zero as $n, m \rightarrow \infty$. Furthermore, since $P_{n} u \rightarrow u$ for any $u$ in $X$ and $g_{n} \rightarrow g$ as $n \rightarrow \infty$, the other terms also go to zero as $n, m \rightarrow \infty$. Thus $\left\{g_{n}^{\prime}\right\}=\left\{P_{n} A^{\prime}\left(x_{0}\right) x_{n}-p x_{n}\right\}$ is a Cauchy sequence and therefore, by $P$ compactness of $A^{\prime}\left(x_{0}\right)$, there exists a subsequence $\left\{x_{n_{1}}\right\}$ and an element $x$ in $X$ such that $x_{n_{i}} \rightarrow x$. By the continuity of $A, P_{n_{i}} A x_{n_{i}} \rightarrow A x$. Consequently, $A$ is $P$-compact.

Converse. Suppose now that $A$ is $P$-compact and $x$ is any given point in $X$. Suppose further that for any bounded sequence $\left\{x_{n} \mid x_{n} \in X_{n}\right\}$ and some $p>0$

$$
g_{n}=P_{n} A^{\prime}(x) x_{n}-p x_{n} \rightarrow g \quad \text { as } n \rightarrow \infty \text {. }
$$

First, by definition of the Fréchet derivative, $A^{\prime}(x)$ is a bounded linear operator and, therefore, $P_{n} A^{\prime}(x)$ is continuous in $X_{n}$ for each $n$. Without loss of generality we may assume that $\left\{x_{n}\right\}$ converges weakly to some element $x_{0}$ in $X$. Now put $z_{n}=P_{n} x+x_{n}-P_{n} x_{0}$ and observe that $z_{n} \in X_{n}$ and $z_{n} \rightarrow x$ as $n \rightarrow \infty$. Choose a unit vector $e_{n m}$ in $X^{*}$ such that for $\tilde{g}_{n}=P_{n} A z_{n}-p z_{n}$ we have $\left\|\tilde{g}_{n}-\tilde{g}_{m}\right\|=\left(\tilde{g}_{n}-\tilde{g}_{m}, e_{n m}\right)$. Thus adding and subtracting $P_{n} A x$ and $P_{m} A x$ we get

$$
\begin{aligned}
\left\|\tilde{g}_{n}-\tilde{g}_{m}\right\|= & \left(\left\{P_{n} A z_{n}-P_{n} A x-p z_{n}\right\}-\left\{P_{m} A z_{m}-P_{m} A x-p z_{m}\right\}, e_{n m}\right) \\
& +\left(\left\{P_{n} A x-P_{m} A x\right\}, e_{n m}\right) .
\end{aligned}
$$

As in the first part of the proof, by the mean-value theorem, there exists $t_{n m}$ and $t_{n m}^{\prime}$ in $(0,1)$ such that

$$
\begin{aligned}
\left\|\tilde{g}_{n}-\tilde{g}_{m}\right\|= & \left(\left\{P_{n} A^{\prime}\left(u_{n m}\right)\left(z_{n}-x\right)-p z_{n}\right\}-\left\{P_{m} A^{\prime}\left(u_{n m}^{\prime}\right)\left(z_{m}-x\right)-p z_{m}\right\}, e_{n m}\right) \\
& +\left(\left\{P_{n} A x-P_{m} A x\right\}, e_{n m}\right),
\end{aligned}
$$

where we have put $u_{n m}=x+t_{n m}\left(z_{n}-x\right)$ and $u_{n m}^{\prime}=x+t_{n m}^{\prime}\left(z_{m}-x\right)$. Now adding and subtracting $P_{n} A^{\prime}(x) z_{n}$ and $P_{m} A^{\prime}(x) z_{m}$ to the right-hand side of the above equality we obtain

$$
\begin{aligned}
\left\|\tilde{g}_{n}-\tilde{g}_{m}\right\|= & \left(\left\{P_{n} A^{\prime}(x) z_{n}-p z_{n}\right\}-\left\{P_{m} A^{\prime}(x) z_{m}-p z_{m}\right\}, e_{n m}\right) \\
& -\left(\left\{P_{n} A^{\prime}(x) x-P_{m} A^{\prime}(x) x\right\}, e_{n m}\right)+\left(\left\{P_{n} A^{\prime}\left(u_{n m}\right)-P_{n} A^{\prime}(x)\right\}\left(z_{n}-x\right), e_{n m}\right) \\
& +\left(\left\{P_{m} A^{\prime}(x)-P_{m} A^{\prime}\left(u_{n m}^{\prime}\right)\right\}\left(z_{m}-x\right), e_{n m}\right)+\left(\left\{P_{n} A x-P_{m} A x\right\}, e_{n m}\right) .
\end{aligned}
$$

Since $z_{n}=P_{n} x+x_{n}-P_{n} x_{0}$ and $g_{n}=P_{n} A^{\prime}(x) x_{n}-p x_{n}$ the last equality yields the lookedfor relation

$$
\begin{aligned}
\left\|\tilde{g}_{n}-\tilde{g}_{m}\right\|= & \left(g_{n}-g_{m}, e_{n m}\right)+\left(\left\{P_{n} A^{\prime}(x) P_{n} x-P_{m} A^{\prime}(x) P_{m} x\right\}, e_{n m}\right) \\
& +\left(\left\{P_{m} A^{\prime}(x) P_{m} x_{0}-P_{n} A^{\prime}(x) P_{n} x_{0}\right\}, e_{n m}\right)+p\left(P_{m} x-P_{n} x+P_{n} x_{0}-P_{m} x_{0}, e_{n m}\right) \\
& +\left(\left\{P_{m} A^{\prime}(x) x-P_{n} A^{\prime}(x) x\right\}, e_{n m}\right)+\left(\left\{P_{n} A x-P_{m} A x\right\}, e_{n m}\right) \\
& +\left(\left\{P_{n} A^{\prime}\left(u_{n m}\right)-P_{n} A^{\prime}(x)\right\}\left(z_{n}-x\right), e_{n m}\right)+\left(\left\{P_{m} A^{\prime}(x)-P_{m} A^{\prime}\left(u_{n m}^{\prime}\right)\right\}\left(z_{m}-x\right), e_{n m}\right) .
\end{aligned}
$$


Since $g_{n} \rightarrow g, P_{n} u \rightarrow u$ for each $u \in X, A^{\prime}(x)$ is continuous and $A^{\prime}\left(u_{n m}\right) \rightarrow A^{\prime}(x)$ in $L(X, X)$, it follows from the above equality that $\left\{\tilde{g}_{n}\right\}$ is a Cauchy sequence. Thus, by the $P$-compactness of $A$, there exists a subsequence $\left\{z_{n_{i}}\right\}=\left\{P_{n_{i}} x+x_{n_{i}}-P_{n_{i}} x_{0}\right\}$ and an element $y$ in $X$ such that $z_{n_{i}} \rightarrow y$ and, therefore, $x_{n_{i}}=z_{n_{i}}+P_{n_{i}} x_{0}-P_{n_{i}} x$ $\rightarrow y+x_{0}-x \equiv \tilde{x}$. By the continuity of $A^{\prime}(x), P_{n_{i}} A^{\prime}(x) x_{n_{i}} \rightarrow A(x) \tilde{x}$, i.e., $A^{\prime}(x)$ is $P$-compact.

3. Linear $P$-compact operators. In this section, we discuss the properties of linear $P$-compact mappings of $X$ into $X$. The main result of this section is the characterization theorem which states that if $X$ is a real Hilbert space $H$ and $A$ is a symmetric linear operator of $H$ into $H$, then $A$ is $P$-compact if and only if $A$ is of the form $A=S+T$ where $S$ is nonpositive and $T$ is completely continuous in $H$. We start with the following theorem for linear (not necessarily bounded) operators acting in $X$.

Theorem 3.1. (a) Let $A$ be a linear P-compact mapping of $X$ into $X$ and let $\lambda>0$ be an arbitrary real number. Either the nonhomogeneous equation $\lambda x-A x=y$ has a unique solution $x$ in $X$ whatever the given element $y$ in $X$ (and in particular has the unique solution $x=0$ when $y=0$ ) or the homogeneous equation $\lambda x-A x=0$ has nonzero solutions.

(b) If, additionally, we assume that $A$ is bounded, then the number of linearly independent solutions of the homogeneous equation is finite.

Proof. Suppose that the homogeneous equation $\lambda x-A x=0$ has $x=0$ as its only solution. Then there exists an integer $N>0$ and a constant $\alpha>0$ such that

$$
\left\|\lambda x-P_{n} A x\right\| \geqq \alpha\|x\| \text { for all } x \text { in } X_{n} \text { and all } n \geqq N \text {. }
$$

If the latter assertion were not true for any $N>0$, then we could find a sequence $\left\{x_{n}\right\}$ with $x_{n} \in X_{n}$ and, in view of the linearity of $A$, such that $\left\|x_{n}\right\|=1$ and

$$
\lambda x_{n}-P_{n} A x_{n} \rightarrow 0 \text { as } n \rightarrow \infty .
$$

But, by the $P$-compactness of $A$, this implies the existence of a subsequence $\left\{x_{n_{i}}\right\}$ and an element $x$ in $X$ such that $x_{n} \rightarrow x$ and $P_{n_{i}} A x_{n_{i}} \rightarrow A x$ as $n_{i} \rightarrow \infty$. Hence, $\|x\|=1$ and $\lambda x-A x=0$ contradicting the fact that $\lambda x-A x=0$ has only a trivial solution. Thus, for $n \geqq N, \lambda I-P_{n} A$ are one-to-one finite-dimensional linear operators and, therefore, are onto. Hence, for any given $y$ in $X$, there exists a unique $x_{n}$ in $X_{n}$ such that $\lambda x_{n}-P_{n} A x_{n}=P_{n} y$. Now, since $\alpha\left\|x_{n}\right\| \leqq\left\|\lambda x_{n}-P_{n} A x_{n}\right\|=\left\|P_{n} y\right\|$ $\leqq K\|y\|$ and $P_{n} y \rightarrow y$ as $n \rightarrow \infty$, it follows that $\left\{x_{n}\right\}$ with $x_{n} \in X_{n}$ is bounded. Thus, the $P$-compactness of $A$ implies the existence of a subsequence $\left\{x_{n_{1}}\right\}$ and an element $x$ in $X$ such that $x_{n_{i}} \rightarrow x$ and $P_{n_{i}} A x_{n_{i}} \rightarrow A x$ as $n_{i} \rightarrow \infty$. This and our assumption imply that $x$ is the unique solution of the nonhomogeneous equation.

To prove assertion (b), suppose now that $A$ is bounded and that the null space, $N(\lambda I-A)$, contains nonzero elements. Then we claim that $N(\lambda I-A)$ is finitedimensional. If this were not the case, then because the sphere $\|x\|=1$ in the infinite 
dimensional space $N(\lambda I-A)$ is not compact there exists a sequence of elements $x_{1}, x_{2}, x_{3}, \ldots\left(\left\|x_{k}\right\|=1\right)$ in $N(\lambda I-A)$ such that $\left\|x_{m}-x_{n}\right\|>1 / 2$ for $m \neq n$. Since the elements $x$ of $N(\lambda I-A)$ are characterized by the relation $\lambda x-A x=0$ we have $\lambda x_{k}-A x_{k}=0$ for $k=1,2, \ldots$ Now, since the system $\left(\left\{X_{n}\right\},\left\{P_{n}\right\}\right)$ is projectionally complete in $X$, for each $k$ and $\varepsilon_{k}=1 / k$, there exists an integer $n(k)$ (which we can and shall assume that $n(k)>k)$ such that $\left\|x_{k}-P_{n(k)} x_{k}\right\|<\varepsilon_{k}$. This, the boundedness of $A$ and the equality $\lambda x_{k}-A x_{k}=0$ imply that with $w_{n(k)}=P_{n(k)} x_{k}$ in $X_{n(k)}$, we have

$$
\begin{aligned}
\left\|P_{n(k)} A w_{n(k)}-\lambda w_{n(k)}\right\| & \leqq\left\|P_{n(k)} A w_{n(k)}-P_{n(k)} A x_{k}\right\|+\left\|P_{n(k)} A x_{k}-\lambda w_{n(k)}\right\| \\
& \leqq K\|A\|\left\|w_{n(k)}-x_{k}\right\|+K\left\|A x_{k}-\lambda x_{k}\right\| \rightarrow 0 \text { as } k \rightarrow \infty .
\end{aligned}
$$

Thus, by the $P$-compactness of $A$, there exists a subsequence $\left\{w_{n(m)}\right\}$ of $\left\{w_{n(k)}\right\}$ and an element $w$ in $X$ such that $w_{n(m)} \rightarrow w$ and $P_{n(m)} A w_{n(m)} \rightarrow A w$ as $m \rightarrow \infty$. But $\left\|x_{m}-w\right\| \leqq\left\|x_{m}-w_{n(m)}\right\|+\left\|w_{n(m)}-w\right\| \rightarrow 0$ as $m \rightarrow \infty$. Thus, $\left\{x_{m}\right\}$ is a convergent subsequence, contradicting the fact that $\left\|x_{k}-x_{n}\right\|>1 / 2$ for $n \neq k$. Hence $N(\lambda I-A)$ is finite-dimensional.

Corollary 3.1 (LaX-Milgram Lemma). If $A$ is a bounded linear operator of $H$ into $H$ such that $(A x, x) \geqq c\|x\|^{2}$ for all $x$ in $H$ and some constant $c>0$, then $A$ is one-to-one and onto.

Proof. Since $A$ is linear and $B=c I-A$ is such that $(B x, x)=c(x, x)-(A x, x) \leqq 0$, Corollary 2.1 implies that $B$ is $P$-compact. Let $\lambda>0$ be any fixed real number and suppose that $\lambda x-B x=0$. Then $0=((\lambda I-B) x, x)=\lambda(x, x)-(B x, x) \geqq \lambda\|x\|^{2}$ implying that $x=0$. Hence, by Theorem 3.1, the operator $(\lambda I-B)$ is one-to-one and onto for any given $\lambda>0$. In particular, for $\lambda=c$ the operator $(c I-B)=A$ is one-to-one and onto.

Our next result is the following interesting theorem which plays an essential role in the characterization of symmetric $P$-compact operators.

THEOREM 3.2. If $A$ is a symmetric nonnegative (i.e, $A=A^{*}$ and $A \geqq 0$ ) P-compact operator of $H$ into $H$, then $A$ is completely continuous.

Proof. The proof of Theorem 3.2 ensues from the following lemmas.

LEMMA 3.1. Under the assumptions of Theorem 3.2, the number $\mu=\|A\|$ is an eigenvalue of $A$, and the corresponding eigenspace $H_{\mu}$ is finite-dimensional.

Proof. Consider the finite-dimensional operators $A_{n}=P_{n} A P_{n}$. They are symmetric and nonnegative as mappings of $H_{n}$ into $H_{n}$. Hence for each $n$ there exists an $x_{n} \in H_{n}$ with $\left\|x_{n}\right\|=1$ such that

$$
A_{n} x_{n}=\mu_{n} x_{n}, \quad \mu_{n}=\sup _{x \in H_{n}} \frac{\left\|A_{n} x\right\|}{\|x\|} .
$$

It is obvious that for each $n, 0 \leqq \mu_{n} \leqq \mu$ where $\mu=\sup _{x \in H}\|A x\| /\|x\|$. Let $\tilde{\mu}$ be the 
1.u.b. of $\left\{\mu_{n}\right\}$. Hence there exists a subsequence $\left\{\mu_{m}\right\}$ of $\left\{\mu_{n}\right\}$ such that $\mu_{m} \rightarrow \tilde{\mu}$ and $\tilde{\mu} \leqq \mu$. It follows from (3.1) that

$$
A_{m} x_{m}-\tilde{\mu} x_{m}=\left(\mu_{m}-\tilde{\mu}\right) x_{m} \rightarrow 0 \text { as } m \rightarrow \infty .
$$

Since $A$ is $P$-compact, there exists a subsequence $\left\{x_{m_{i}}\right\}$ and an element $\tilde{x}$ in $H$ such that $x_{m_{i}} \rightarrow \tilde{x}$ and $A_{m_{i}} x_{m_{i}} \rightarrow A \tilde{x}$ as $m_{i} \rightarrow \infty$. Hence (3.2) implies that $A \tilde{x}-\tilde{\mu} \tilde{x}=0$, i.e., $\tilde{\mu}$ is an eigenvalue of $A$ and $\tilde{x}$ is its corresponding eigenvector with $\|\tilde{x}\|=1$. To prove that $\tilde{\mu}=\mu$ note that $A_{n} x \rightarrow A x$ for each $x$ in $H$ and that for each $x$ in $H$ and each $n$, with $P_{n} x=y_{n}$, we have

$$
\left\|A_{n} y_{n}\right\| \leqq \tilde{\mu}\left\|y_{n}\right\| \text { or }\left\|A_{n} x\right\| \leqq \tilde{\mu}\left\|P_{n} x\right\| .
$$

Passing to the limit we obtain $\|A x\| \leqq \tilde{\mu}\|x\|$. This implies that $\tilde{\mu} \geqq \mu$ and hence $\tilde{\mu}=\mu$.

The finite dimensionality of the eigenspace $H_{\mu}$ follows from assertion (b) of Theorem 3.1.

Now set $\bar{H}=H \ominus H_{\mu}$ and denote $\left.\bar{A} \equiv A\right|_{\bar{H}}$. It is clear that $A: H_{\mu} \rightarrow H_{\mu}$, and since $A$ is symmetric, it is also clear that $A: \bar{H} \rightarrow \bar{H}$. Thus, $A$ is reduced by $H_{\mu}$, and we have the standard result [35]:

Lemma 3.2. (a) If $\bar{P}$ and $P_{\mu}$ are projections of $H$ onto $\bar{H}$ and $H_{\mu}$ respectively, then $H_{\mu}$ reduces $A$ if and only if $P_{\mu} A=A P_{\mu}$ and $\bar{P} A=A \bar{P}$.

(b) If $H_{\mu}$ reduces $A$, then it reduces $\lambda I-A$.

(c) If $H_{\mu}$ reduces $A$, and if $\bar{A}$ and $A_{\mu}$ are restrictions of $A$ to $\bar{H}$ and $H_{\mu}$ respectively, then $R(A)=R(\bar{A}) \oplus R\left(A_{\mu}\right) ; A^{-1}$ exists if and only if $\bar{A}^{-1}$ and $A_{\mu}^{-1}$ exist; $\sigma(A)$ $=\sigma(\bar{A}) \cup \sigma\left(A_{\mu}\right) ;$ and $p \sigma(A)=p \sigma(\bar{A}) \cup p \sigma\left(A_{\mu}\right)$ where $p \sigma(A)$ denotes the point spectrum of $A$.

Thus, $A$ can be represented as $A=\bar{A} \oplus A_{\mu}$, and any $x \in H$ has a unique representation as $x=\bar{x}+x_{\mu}$ with $\bar{x} \in \bar{H}, x_{\mu} \in H_{\mu}$. Furthermore, for any $x \in H, A x=$ $A \bar{x}+A x_{\mu}=\bar{A} \bar{x}+A_{\mu} x_{\mu}$. We wish to prove that $\bar{A}$ is a symmetric, nonnegative $P$-compact operator on $\bar{H}$. This will follow from the following general result.

Lemma 3.3. If $A$ is a symmetric nonnegative P-compact operator on $H, H=$ $\bar{H} \oplus \tilde{H}$, where $\tilde{H}$ reduces $A, \bar{A}=\left.A\right|_{\bar{H}}$, then $\bar{A}$ is a symmetric nonnegative $P$-compact operator on $\bar{H}$.

Proof. Symmetry and nonnegativeness are obvious. Let $\left(\left\{\bar{H}_{n}\right\},\left\{\bar{P}_{n}\right\}\right)$ and $\left(\left\{\tilde{H}_{n}\right\},\left\{\tilde{P}_{n}\right\}\right)$ be projectionally complete in $\bar{H}$ and $\tilde{H}$, respectively. Suppose that $\left\{\bar{x}_{n}\right\}$ is a bounded sequence in $\bar{H}$ with $\bar{x}_{n} \in \bar{H}_{n}$ so that for some $p>0$,

$$
\bar{P}_{n} \bar{A} \bar{x}_{n}-p \bar{x}_{n} \rightarrow \bar{g} \in \bar{H} \text {. }
$$

Now put $H_{n}=\bar{H}_{n} \oplus \tilde{H}_{n}$ and $P_{n}=\bar{P}_{n} \oplus \tilde{P}_{n}$. It is clear that $\left(\left\{H_{n}\right\},\left\{P_{n}\right\}\right)$ is projectionally complete in $H$. Furthermore, let $\tilde{x}$ be any element in $\tilde{H}$, and set $x_{n}=\bar{x}_{n}+\widetilde{P}_{n} \tilde{x}$. Then $\left\{x_{n}\right\}$ is a bounded set in $H$ with $x_{n} \in H_{n}$, and

$$
\begin{aligned}
P_{n} A x_{n}-p x_{n} & =P_{n} A\left(\bar{x}_{n}+\tilde{P}_{n} \tilde{x}\right)-p\left(\bar{x}_{n}+\tilde{P}_{n} \tilde{x}\right) \\
& =P_{n} A \bar{x}_{n}-p \bar{x}_{n}+P_{n} A \widetilde{P}_{n} \tilde{x}-p \tilde{P}_{n} \tilde{x} \\
& =\bar{P}_{n} \bar{A} \bar{x}_{n}-p \bar{x}_{n}+P_{n} A \tilde{P}_{n} \tilde{x}-p \tilde{P}_{n} \tilde{x} \rightarrow g+A \tilde{x}-p \tilde{x} .
\end{aligned}
$$


Since $A$ is $P$-compact, there exists a subsequence $\left\{x_{n_{i}}\right\}$ with $x_{n_{i}}=\bar{x}_{n_{i}}+\widetilde{P}_{n_{i}} \tilde{x}$ and an element $x \in H$ such that $x_{n_{i}} \rightarrow x$ and $P_{n_{i}} A x_{n_{i}} \rightarrow A x$. Therefore $\bar{x}_{n_{i}} \rightarrow x-\tilde{x}=\bar{x} \in \bar{H}$, and $\bar{P}_{n_{i}} \bar{A} \bar{x}_{n_{i}} \rightarrow \bar{A} \bar{x}$, and hence, $\bar{A}$ is a $P$-compact mapping of $\bar{H}$ into $\bar{H}$.

Proof of Theorem 3.2. By Lemma 3.3, we can apply Lemma 3.1 to $\bar{A}$. Hence there exists an eigenvalue $\bar{\mu}<\mu$ of $\bar{A}$ and a corresponding finite dimensional eigenspace $H \bar{\mu}$. Repeating the arguments above, this procedure furnishes a monotonically decreasing sequence of positive numbers

$$
\mu=\mu_{1}, \quad \bar{\mu}=\mu_{2}, \ldots, \mu_{n}, \ldots,
$$

where each $\mu_{i}$ appears as many times as its multiplicity. Thus, there exists a $\mu_{0} \geqq 0$ such that $\mu_{i} \rightarrow \mu_{0}$. We claim that $\mu_{0}=0$. For suppose $\mu_{0}>0$, and let $\left\{x_{i}\right\}$ be an orthonormal set of eigenvectors corresponding to $\left\{\mu_{i}\right\}$. Then constructing the set $\left\{w_{n(i)}\right\}$ as in Theorem 3.1, we have

$$
\begin{aligned}
\left\|P_{n(i)} A w_{n(i)}-\mu_{0} w_{n(i)}\right\| \leqq & \left\|P_{n(i)} A w_{n(i)}-P_{n(i)} A x_{i}\right\|+\cdots \\
& +\left\|P_{n(i)} A x_{i}-\mu_{i} P_{n(i)} x_{i}\right\|+\left\|\mu_{i} P_{n(i)} x_{i}-\mu_{0} w_{n(i)}\right\| \\
\leqq & \|A\|\left\|w_{n(i)}-x_{i}\right\|+\left\|A x_{i}-\mu_{i} x_{i}\right\|+\left|\mu_{i}-\mu_{0}\right|\left\|x_{i}\right\| \rightarrow 0 .
\end{aligned}
$$

Thus, as in the proof of Theorem 3.1, there exists a convergent subsequence $\left\{x_{i,}\right\}$ which contradicts the orthonormality of the $\left\{x_{i}\right\}$. Hence $\mu_{0}=0$ and since, as is not hard to see, $A x=\sum_{i=1}^{\infty} \mu_{i}\left(x, x_{i}\right) x_{i}$ for each $x$ in $H$, it follows (see Riesz-Sz. Nagy [31]) that $A$ is completely continuous.

The main result of this section is the following theorem:

Theorem 3.3. Let $A$ be a symmetric linear mapping of $H$ into $H$. Then $A$ is $P$ compact if and only if $A$ can be written in the form $A=S+T$, where $S \leqq 0$ and $T$ is completely continuous.

Proof. Suppose $A=S+T$ where $S \leqq 0$ and $T$ is completely continuous. Then, by Corollary $2.1, S$ is $P$-compact and, by part (f) of Lemma $2.1, A=S+T$ is $P$-compact.

Converse. Suppose $A$ is a symmetric $P$-compact operator. Let $E_{0}$ be the orthogonal projection of $H$ onto the eigenspace $H_{0}$, the space corresponding to the nonpositive eigenvalues of $A$. It is clear that $H_{0}$ reduces $A$, and $A=A E_{0}+A\left(I-E_{0}\right)$, where $A E_{0} \leqq 0$ and $A\left(I-E_{0}\right) \geqq 0$. By Lemma 3.3, $T=A\left(I-E_{0}\right)$ is $P$-compact, so by Theorem 3.2, $T$ is completely continuous. Thus, setting $S=A E_{0}, A=S+T$ with $S \leqq 0$ and $T$ completely continuous.

4. Fixed point theorems for generalized $P$-compact operators. The main purpose of this section is to generalize two major fixed point theorems established by Petryshyn for bounded $P$-compact operators $A$ in [23], [24] and for unbounded $P$-compact operators $A$ in [25]. Our extensions consist in showing that under the same conditions as in [24], [25] the corresponding fixed point theorems remain valid for the $P_{\gamma}$-mappings $A$ which map an arbitrary bounded, closed convex set 
$D(A)=C$ with an interior into $X$ and which satisfy appropriate conditions on the boundary $\partial C$ of $C$. Thus in our present case $A$ need not be defined on the entire Banach space $X$. The proofs of the main results follow the arguments of Yamamuro [40] in the finite-dimensional case and those of [24], [25] in the infinite dimensional case. First, we shall prove the generalized theorems under the assumption that $0 \in \operatorname{int} C$.

THEOREM 4.1. Let $A$ be a continuous mapping of a bounded, closed, convex set $C$ of the finite dimensional Banach space $X$ into $X$. Suppose that $0 \in$ int $C$ and for some $\mu>0, A$ satisfies the condition:

$\left(\pi_{\mu}^{<}\right)$: If for some $x \in \partial C$ the equation $A x=\alpha x$ holds then $\alpha<\mu$.

Then there exists at least one element $u$ in $(C-\partial C)$ such that $A u-\mu u=0$.

Proof. For any $t \in[0,1]$ consider the continuous homotopy mapping

$$
A_{t}=t\{\mu I-A\}+\mu(1-t) I=\mu I-t A .
$$

Then it is clear that $A_{t} x \neq 0$ for all $x$ in $\partial C$ and all $t \in[0,1]$. For suppose there were a $t_{0}$ in $[0,1]$ and an $x_{0}$ in $\partial C$ such that $A_{t_{0}} x_{0}=0$. Then we would have $t_{0} A x_{0}=\mu x_{0}$. If $t_{0}=0$, then $x_{0}=0$, since $\mu>0$, contradicting the hypothesis that $0 \in$ int $C$. On the other hand, if $t_{0}>0$, then $A x_{0}=\left(\mu / t_{0}\right) x_{0}$ with $\mu / t_{0} \geqq \mu$, contradicting the hypothesis that $A$ satisfies the condition $\left(\pi_{\mu}^{<}\right)$on $\partial C$. Thus, the degree $\left({ }^{5}\right)$ of $A_{t}$ at 0 , as a mapping in $C$, is constant for $0 \leqq t \leqq 1$. Since the degree of $A_{0}=\mu I$ is one, it follows that the degree of $A_{1}=\mu I-A$ is also one. Therefore, there exists at least one element $u \in(C-\partial C)$ such that $A_{1} u=\mu u-A u=0$.

In view of the fact that Theorem 1 in [25] is an extension of Theorem 2 in [24], to generalize both theorems to the set $C$, it suffices to generalize Theorem 1 .

THeOREM 4.2. Suppose that $A$ is a $P_{\gamma}$-compact mapping of a bounded closed convex set $D(A)=C$ of the Banach space $X$ into $X$. Suppose further that $0 \in$ int $C$ and that, for some $\mu$ dominating $\gamma$ (i.e., $\mu \geqq \gamma$ if $\gamma>0$ and $\mu>\gamma$ if $\gamma=0$ ), $A$ satisfies both of the following conditions:

$(\Lambda):$ There exists a number $c>0$ such that if for any $n, P_{n} A x=\lambda x$ holds for $x \in \partial C$ then $\lambda \leqq c$.

$\left(\pi_{\mu}^{<}\right)$: If for some $x$ in $\partial C$ the equation $A x=\alpha x$ holds then $\alpha<\mu$.

Then there exists at least one element $u$ in $(C-\partial C)$ such that $A u-\mu u=0$.

Proof. The proof of Theorem 4.2 follows the same line of argument as that of Theorem 1 in [25]. All we need to show is that the present conditions $(\Lambda)$ and $\left(\pi_{\mu}^{<}\right)$imply the validity of the following lemma.

Lemma 4.1. If $A$ satisfies the conditions of Theorem 4.2, then there exists an integer $n_{0}>0$ such that if $n \geqq n_{0}$ and $P_{n} A x=\beta x$ for some $x$ in $X_{n} \cap \partial C$, then $\beta<\mu$.

$\left({ }^{5}\right)$ For the definition and the properties of the "degree" of a mapping, see [19]. 
Proof of Lemma 4.1. If the assertion of Lemma 4.1 were not true for any $n_{0}$, we could find a sequence $\left\{x_{n}\right\}$ with $x_{n} \in X_{n} \cap \partial C$ and a sequence of numbers $\left\{\beta_{n}\right\}$ such that

$$
P_{n} A x_{n}=\beta_{n} x_{n}, \quad\left(\beta_{n} \geqq \mu\right) .
$$

Hence our condition $(\Lambda)$ implies that $\beta_{n} \leqq c$, i.e., $\beta_{n} \in[\mu, c]$ for each $n$. Passing to a subsequence, we may assume that $\beta_{n} \rightarrow \beta$ and $\beta \in[\mu, c]$. This, (4.1), and the boundedness of $C$ imply that

$$
P_{n} A x_{n}-\beta x_{n}=\left(\beta_{n}-\beta\right) x_{n} \rightarrow 0 \text { as } n \rightarrow \infty \text {. }
$$

Since $A$ is $P_{\gamma}$-compact and $\beta$ dominates $\gamma,(4.2)$ implies the existence of a strongly convergent subsequence $\left\{x_{n_{1}}\right\}$ of $\left\{x_{n}\right\}$ and an element $x$ in $\partial C$ such that $x_{n_{i}} \rightarrow x$ and $P_{n_{i}} A x_{n_{i}} \rightarrow A x$. This implies that $A x-\beta x=0$ for $x \in \partial C$ and $\beta \geqq \mu$ in contradiction to condition $\left(\pi_{\mu}^{<}\right)$.

To complete the proof of Theorem 4.2, we see from Lemma 4.1 that there exists an integer $n_{1}>0$ such that for all $n \geqq n_{1}, P_{n} A$ is continuous in $X_{n} \cap C$ and satisfies the condition $\left(\pi_{\mu}^{<}\right)$on $X_{n} \cap \partial C$. Since $0 \in$ int $C \cap X_{n}$ for all $n$, Theorem 4.1 implies the existence of at least one element $u_{n}$ in $(C-\partial C) \cap X_{n}$ such that $P_{n} A u_{n}$ $-\mu u_{n}=0$. By $P_{\gamma}$-compactness of $A$, there exists a subsequence $\left\{u_{n_{1}}\right\}$ and an element $u$ in $C$ such that $u_{n_{i}} \rightarrow u$ and $P_{n_{i}} A u_{n_{i}} \rightarrow A u$ as $n_{i} \rightarrow \infty$ and $A u-\mu u=0$. Note that the validity of the last equation implies that $u \in(C-\partial C)$, for the assumption that $u \in \partial C$ would lead to a contradiction of the condition $\left(\pi_{\mu}^{<}\right)$.

REMARK 4.1. The condition $(\Lambda)$ of Theorem 4.2 is, for example, satisfied when $A$ is either bounded or $A(\partial C) \subset C$. Indeed if $A$ is bounded, then $\left\|P_{n} A x\right\| \leqq M$ for some $M>0$ and all $x$ in $\partial C$; therefore, if $P_{n} A x=\lambda x$ for any $n$ and $x$ in $\partial C$, then since the conditions $x \in \partial C$ and $0 \in$ int $C$ imply that $\|x\| \geqq \delta$ for some $\delta>0$, it follows that $\lambda \leqq M / \delta$ because $\delta|\lambda| \leqq|\lambda|\|x\|=\left\|P_{n} A x\right\| \leqq M$. On the other hand, if $A(\partial C) \subset C$, then denoting the diameter of $C$ by $d(C)=\sup _{x, y \in C}\|x-y\|$ we see that, since $A x \in C$ for any $x \in \partial C, \delta|\lambda| \leqq\|\lambda x\|=\left\|P_{n} A x\right\| \leqq K\|A x\| \leqq K d(C)$ from which our assertion follows.

An immediate consequence of Theorem 4.2 is the following general result asserting the existence of fixed points.

TheOREM 4.3. Suppose that $A$ is a $P_{\gamma}$-compact mapping of $C$ (with $0 \in$ int $C$ ) into $X$ for $\gamma \leqq 1$ such that $A$ satisfies conditions $(\Lambda)$ and $\left(\pi_{1}^{<}\right)$on $\partial C$. Then $A$ has at least one fixed point in $(C-\partial C)$.

Corollary 4.1. Suppose $A$ is a $P_{\gamma}$-compact mapping of $C(0 \in \operatorname{int} C)$ into $X$ for $\gamma \leqq 1$. Then $A$ has a fixed point in $C$ provided

(1) $A(\partial C) \subset C$ (and, in particular, $A(C) \subset C$ ) or

(2) $A$ is bounded and $\|A x-x\|^{2} \geqq\|A x\|^{2}-\|x\|^{2}$ for all $x$ in $\partial C$.

Proof. In view of Theorem 4.3 and Remark 4.1, to prove Corollary 4.1 it suffices to show that (1), as well as (2) implies condition $\left(\pi_{1}^{<}\right)$on $\partial C$. 
First, we assume without loss of generality that $A$ has no fixed points on $\partial C$. Suppose now that $A(\partial C) \subset C$ and $A x=\alpha x$ for some $x$ in $\partial C$. Since we want to show that $\alpha<1$ we may assume that $\alpha \geqq 0$. Hence, since by assumption $A x \in C$, and $A x=\alpha x$, it follows that $\alpha<1$, i.e., (1) implies $\left(\pi_{1}^{<}\right)$.

To prove that (2) implies ( $\left.\pi_{1}^{<}\right)$, suppose $A x=\alpha x$ for some $x$ in $\partial C$. Then by (2), $(\alpha-1)^{2} \geqq \alpha^{2}-1$ or $\alpha<1$.

In the next theorem, we drop the condition that $0 \in \operatorname{int} C$ but assume instead that $C$ has an interior and employ Theorem 4.2 in the proof of the following main fixed point theorem of this paper.

THEOREM 4.4. Suppose that $A$ is a $P_{\gamma}$-compact mapping of a bounded closed convex set $C$ (with an interior) into $X$. Suppose further that for some $\mu$ dominating $\gamma$ the operator A satisfies both of the following conditions:

$(\tilde{\Lambda}):$ There exists $a$ constant $c>0$ such that if for some $x_{0} \in \operatorname{int} C$ and any $n$ the equation $P_{n} A y-P_{n} x_{0}=\lambda\left(y-x_{0}\right)$ holds for $y$ in $\partial C$, then $\lambda \leqq c$.

$\left(\tilde{\pi}_{\mu}^{<}\right)$: If for some $x$ in $\partial C$ the equation $A x-x_{0}=\alpha\left(x-x_{0}\right)$ holds, then $\alpha<\mu$.

Then there exists at least one element $u$ in $(C-\partial C)$ such that $A u-x_{0}=\mu\left(x-x_{0}\right)$.

Proof. Consider the set $C^{\prime}=C-x_{0}=\left\{x-x_{0} \mid x \in C\right\}$. It is easy to see that $C^{\prime}$ is a bounded closed convex set in $X$ with $0 \in$ int $C^{\prime}$ and $\partial C^{\prime}=\partial C-x_{0}$. Now define the mapping $A^{\prime}\left(y^{\prime}\right)$ for $y^{\prime}$ in $C^{\prime}$ and $y^{\prime}=\left\{x-x_{0} \mid x \in C\right\}$ by $A^{\prime}\left(y^{\prime}\right)=A x-x_{0}$. Then $A^{\prime}$ maps $C^{\prime}$ into $X$ and $A^{\prime}$ is $P_{\gamma}$-compact. Furthermore, if $P_{n} A^{\prime} y^{\prime}=\lambda y^{\prime}$ for $y^{\prime}$ in $\partial C^{\prime}$, then $\lambda \leqq c$ by $(\tilde{\Lambda})$ since $P_{n} A^{\prime} y^{\prime}=P_{n} A x-P_{n} x_{0}=\lambda\left(x-x_{0}\right)$. Also, if $A^{\prime}\left(y^{\prime}\right)=\alpha y^{\prime}$ for some $y^{\prime} \in \partial C^{\prime}$, then by $\left(\tilde{\pi}_{\mu}^{<}\right), \alpha<\mu$ since $A^{\prime}\left(y^{\prime}\right)=A x-x_{0}=\alpha\left(x-x_{0}\right)$. Thus, $A^{\prime}$ and $C^{\prime}$ satisfy all the conditions of Theorem 4.2. Hence there exists an element $u^{\prime} \in\left(C^{\prime}-\partial C^{\prime}\right)$ such that $A^{\prime}\left(u^{\prime}\right)-\mu u^{\prime}=0$, or $A u-x_{0}=\mu\left(u-x_{0}\right)$ with $u \in(C-\partial C)$.

THEOREM 4.5. If $A$ is a $P_{\gamma}$-compact operator for $\gamma \leqq 1$ satisfying conditions $(\tilde{\Lambda})$ and $\left(\tilde{\pi}_{1}^{<}\right)$on $\partial C$ of Theorem 4.4 , then $A$ has a fixed point in $(C-\partial C)$.

Remark 4.2. In case $A$ is completely continuous, Theorem 4.5 was proved by Yamamuro [40] by employing the theory of the degree of a mapping.

A number of known fixed point theorems are deducible from the following corollary to Theorem 4.5 .

COROLlaRY 4.2. If $A$ is a $P_{\gamma}$-compact mapping of $C$ (with an interior) into $X$ for $\gamma \leqq 1$, then $A$ has a fixed point in $C$ provided

(1) $A(\partial C) \subset C$ (and, in particular, $A(C) \subset C$ ) or

(2) $A$ is bounded and for some $x_{0}$ in int $C$,

$$
\|A x-x\|^{2} \geqq\left\|A x-x_{0}\right\|^{2}-\left\|x-x_{0}\right\|^{2} \text { for all } x \text { in } \partial C .
$$

Proof. In virtue of Theorem 4.5 it suffices to show that each of our conditions (1) and (2) implies conditions $(\tilde{\Lambda})$ and $\left(\tilde{\pi}_{1}^{<}\right)$on $\partial C$.

Without loss of generality we may assume that $A$ has no fixed points on $\partial C$. If, as in the proof of Theorem 4.4, we take $C^{\prime}=C-x_{0}=\left\{x-x_{0} \mid x \in C\right\}, \partial C^{\prime}=$ $\partial C-x_{0}$, and define $A^{\prime}\left(y^{\prime}\right)$ for $y^{\prime}$ in $C^{\prime}$ and $y^{\prime}=\left\{x-x_{0} \mid x \in C\right\}$ by $A^{\prime}\left(y^{\prime}\right)=A x-x_{0}$, 
then we see that $0 \in$ int $C^{\prime}, A^{\prime}$ maps $C^{\prime}$ into $C^{\prime}, A^{\prime}$ has no fixed points on $\partial C^{\prime}$ and, for all $y^{\prime}$ in $\partial C^{\prime}, A^{\prime}\left(y^{\prime}\right) \in C^{\prime}$ in case (1) while $\left\|A^{\prime}\left(y^{\prime}\right)-y^{\prime}\right\|^{2} \geqq\left\|A^{\prime}\left(y^{\prime}\right)\right\|^{2}-\left\|y^{\prime}\right\|^{2}$ in case (2). Thus, Corollary 4.2 follows from Corollary 4.1.

SPECIAL FIXED POINT THEOREMS. To indicate the generality of our Theorems 4.4 and 4.5 we show here that for Banach spaces having projectionally complete systems $\left(\left\{X_{n}\right\},\left\{P_{n}\right\}\right)$ many of the known fixed point theorems follow as special cases of our Theorem 4.5. Thus, our results supply elementary and essentially constructive proofs of these theorems.

THEOREM O. Let $A$ be a completely continuous mapping of a bounded closed convex set $C$ (with an interior) into $X$. Then $A$ has a fixed point in $C$ provided $A$ satisfies any one of the following conditions:

(S) (Schauder [34]): $A(C) \subset C$.

(R) (Rothe [32]): $A(\partial C) \subset C$ in case $C=B_{r}(0)$.

(A) (Altman [1]): $\|A x-x\|^{2} \geqq\|A x\|^{2}-\|x\|^{2}$ for all $x$ in $\partial C=\partial B_{r}(0)$.

(T) (Tychonoff [37]): $A x-\lambda x \neq 0$ for any $x$ in $\partial C$ and any $\lambda>1$.

Proof. The proofs of the above theorems follow from Theorem 3 in [24] and Theorem 4.5 above. Let us add that if $X=H$, condition (A) is equivalent to the condition $(A x, x) \leqq\|x\|^{2}$ for all $x$ in $\partial B_{r}(0)$ first used by Krasnoselsky [17].

Theorem Y (Yamamuro). Let $A$ be a completely continuous mapping of a bounded closed convex set $C$ (with an interior) into $X$. The mapping $A$ has a fixed point in $C$ provided that if for some $x_{0}$ in int $C$ the equation $A x-x_{0}=\alpha\left(x-x_{0}\right)$ holds for some $x$ in $\partial C$ then $\alpha<1$.

Proof. Theorem Y follows from Theorem 3 in [24] and Theorem 4.5 above.

Theorem HNRR (Hanani, Netanyahu, Reichaw-Reichbach [12]). Let $A$ be a completely continuous mapping of $B_{r}\left(x_{0}\right)$ into $H$. Then $A$ has a fixed point in $B_{r}\left(x_{0}\right)$ provided $\left(x-x_{0}, A x-x_{0}\right) \leqq r^{2}$ for all $x$ in $\partial B_{r}\left(x_{0}\right)$.

Proof. If $X=H$ and $C=B_{r}\left(x_{0}\right)$, then it is not hard to see that condition (2) of Corollary 4.2 is equivalent in this case to the condition $\left(x-x_{0}, A x-x_{0}\right) \leqq r^{2}$ for all $x$ in $\partial B_{r}\left(x_{0}\right)$. Since a completely continuous operator is bounded and $P$-compact, Theorem NHRR follows from Corollary 4.2.

Theorem A (Petryshyn). If $A$ is a bounded P-compact mapping of $B_{r}(0)$ into $X$ such that $A$ satisfies $\left(\pi_{\mu}^{<}\right)$on $\partial B_{r}(0)$ for some $\mu>0$, then there exists a point $u$ in $\left(B_{r}(0)-\partial B_{r}(0)\right)$ such that $A u-\mu u=0$.

REMARK 4.3. As was shown in [24], a special case of Theorem A is a fixed point theorem for quasicompact mappings established by Kaniel [14].

TheORem B (PetryshyN). If $A$ is a P-compact mapping of $B_{r}(0)$ into $X$ such that $A$ satisfies condition $(\Lambda)$ and $\left(\pi_{\mu}^{<}\right)$on $\partial B_{r}$ for some $\mu>0$, then there exists a point $u$ in $\left(B_{r}-\partial B_{r}\right)$ such that $A u-\mu u=0$. 
Proof. Theorem B, which is an extension of Theorem A, is precisely Theorem 4.4 if we take $C=B_{r}(0)$ and $\gamma=0$.

5. Applications of general fixed point theory to the solution of various equations. In this section we apply the fixed point theorems obtained in the previous section to the solution of various classes of nonlinear equations.

THEOREM 5.1. Suppose $\left\|P_{n}\right\|=1$ for all $n$ and $A=S+T$ maps $D(A)=X$ into $X$, where $T$ is completely continuous and $S$ is strictly contractive with Lipschitz constant $L<1$. If on the boundary $\partial C$ of some bounded closed convex set $C$ with an interior the mapping $A$ satisfies condition $\left(\pi_{1}^{<}\right)$, then $A$ has a fixed point in $(C-\partial C)$.

Proof. By. Corollary 2.3, the mapping $S$ is $P_{\gamma}$-compact for any fixed $\gamma>L$, while by Remark 2.1, the map $A=S+T$ is also $P_{\gamma}$-compact. Since $L<1$ and $A$ satisfies $\left(\pi_{1}^{<}\right)$on $\partial C$, the result follows from Theorem 4.5 and Remark 4.1.

REMARK 5.1. In [16] Krasnoselsky showed that if $S$ is a strict contraction on $C$, $T$ is completely continuous and $A=S+T$ is such that

$$
S x+T y \in C \text { for all } x, y \in C,
$$

then $A$ has a fixed point in $C$. We see that, on the one hand, our Theorem 5.1 is weaker than Krasnoselsky's result since unlike him, we impose the conditions on the entire Banach space $X$ while, on the other hand, Theorem 5.1 is stronger than his theorem since our condition $\left(\pi_{1}^{<}\right)$on the boundary $\partial C$ is much weaker and more convenient for applications than the rather strong and restrictive condition $(\mathrm{K})$.

In case $X=H$ or $L<1 / 2$ for $X \neq H$, then we have the following theorem which is stronger than Theorem 2 in [16]. Without loss of generality we assume that $0 \in \operatorname{int} C$.

TheOREM 5.2. Suppose that $A=S+T$. is defined only on $D(A)=C \subset X$ with $0 \in$ int $C$, where $T$ is completely continuous and $S$ is such that for all $x$ and $y$ in $C$

$$
\|S x-S y\| \leqq L\|x-y\|, \quad L<1 .
$$

(a) If $X=H$ and $A$ satisfies $\left(\pi_{1}^{<}\right)$on $\partial C$, then $A$ has a fixed point in $(C-\partial C)$.

(b) If $C=B_{r}(0) \subset X$, and (5.1) holds with $L<1 / 2$ and $\left\|P_{n}\right\|=1$ for all $n$, then $A$ has a fixed point in $C$ provided that $A$ satisfies the condition $\left(\pi_{1}^{<}\right)$on $\partial C=\partial B_{r}$.

Proof (a). Let $R_{C}$ be the retraction of $H$ onto $C$. Then it is not hard to show (see, for example, [7]) that $R_{C}$ is nonexpansive. Thus, if we define the mapping $\tilde{A}$ of $H$ into $H$ by $\tilde{A} x=S R_{C} x+T R_{C} x$ for each $x$ in $H$, then $\tilde{A} x=A x$ for each $x$ in $C, S R_{C}$ is strictly contractive on $H, T R_{C}$ is completely continuous on $H$ and $\tilde{A}$ satisfies $\left(\pi_{1}^{<}\right)$on $\partial C$. Hence, by Theorem 5.1 , there exists a point $x$ in $(C-\partial C)$ such that $\tilde{A} x=A x=x$, i.e., $A$ has a fixed point in $(C-\partial C)$.

(b) Suppose now that $R_{C}$ is the retraction of $X$ onto $C=B_{r}(0)$. Then $R_{C}$ is given by

$$
\begin{aligned}
& R_{C} x=x \quad \text { if }\|x\| \leqq r, \\
& =\frac{r x}{\|x\|} \text { if }\|x\| \geqq r .
\end{aligned}
$$


The proof of this part depends on the following simple lemma:

LEMMA 5.1. The retraction $R_{C}$ of $X$ onto $B_{r}(0)$ given by (5.2) is such that

$$
\left\|R_{C} x-R_{C} y\right\| \leqq 2\|x-y\| \text { for all } x \text { and } y \text { in } X \text {. }
$$

Proof of Lemma 5.1. Lemma 5.1 is certainly true for all $x$ and $y$ in $B_{r}(0)$ while for $x$ and $y$ outside $B_{r}(0)$, i.e., for $\|x\|>r$ and $\|y\|>r$, the inequality

$$
\left\|\frac{x}{\|x\|}-\frac{y}{\|y\|}\right\| \leqq \frac{2}{\max (\|x\|,\|y\|)}\|x-y\|
$$

established in [20] implies that

$$
\left\|R_{C} x-R_{C} y\right\|=r\left\|\frac{x}{\|x\|}-\frac{y}{\|y\|}\right\| \leqq r \cdot \frac{2}{r}\|x-y\|=2\|x-y\| .
$$

Suppose now that $x \in B_{r}(0), y \notin B_{r}(0)$. Then, since $\|y\|>r$,

$$
\begin{aligned}
\left\|R_{C} x-R_{C} y\right\| & =\left\|x-\frac{r y}{\|y\|}\right\| \leqq\|x-y\|+\left\|y-\frac{r y}{\|y\|}\right\| \\
& \leqq\|x-y\|+\left\{1-\frac{r}{\|y\|}\right\}\|y\|=\|x-y\|+\|y\|-r \\
& \leqq\|x-y\|+\|y\|-\|x\| \leqq 2\|x-y\| .
\end{aligned}
$$

Thus we have shown that $\left\|R_{C} x-R_{C} y\right\| \leqq 2\|x-y\|$ for all $x$ and $y$ in $X$.

To complete the proof of part (b) of Theorem 5.2 consider now the mapping $\tilde{A}$ of $X$ into $X$ defined by $\tilde{A} x=S R_{C} x+T R_{C} x$ for all $x$ in $X$. Our conditions imply that $T R_{C}$ is completely continuous and $S R$ is strictly contractive on all of $X$. Consequently, by Corollary 2.3 and Remark 2.1 , the mapping $\tilde{A}$ is $P_{y}$-compact for any fixed $\gamma>2 L$. Furthermore, since $\tilde{A} x=A x$ for all $x$ in $B_{r}, \tilde{A}$ satisfies condition $\left(\pi_{1}^{<}\right)$on $\partial B_{r}$. Hence since $2 L<1$, by Theorem 5.1, $\tilde{A}$ has a fixed point in $\left(B_{r}-\partial B_{r}\right)$, and, thus, $A$ has a fixed point in $\left(B_{r}-\partial B_{r}\right)$.

Theorem 5.3. Let $A$ be a bounded P-compact mapping of $X$ into $X$ such that

$$
\lim _{\|x\| \rightarrow \infty} \frac{\|A x\|}{\|x\|}=0 \text {. }
$$

Then for any given $\lambda>0$ and any given $y$ in $X$ there exists $x$ in $X$ such that

$$
x-\lambda A x=y .
$$

Proof. For an arbitrary but given $\lambda>0$ and $y$ in $X$ consider the mapping $T x$ $=\lambda A x+y$. Clearly $T$ is a bounded $P$-compact operator. Furthermore, there exists a sphere $S_{r}$ such that $T$ satisfies condition $\left(\pi_{1}^{<}\right)$on $S_{r}$. To determine $S_{r}$ suppose that $\alpha x=T x=\lambda A x+y$ for some $x$ in $S_{r}$. Then, assuming without loss of generality that $\alpha \geqq 0$, we have

$$
\alpha\|x\| \leqq \lambda\|A x\|+\|y\| \quad \text { or } \quad \alpha \leqq \lambda \frac{\|A x\|}{\|x\|}+\frac{\|y\|}{\|x\|} .
$$


Since $y$ is fixed, we can choose $r_{0}$ so that $\|y\| /\|x\|<\frac{1}{2}$ for $\|x\| \geqq r_{0}$ while, since $A$ satisfies (5.4) and $\lambda$ is fixed, we can choose $r_{1}>0$ so that $\lambda\|A x\| /\|x\|<\frac{1}{2}$ for $\|x\| \geqq r_{1}$. Thus, for $x$ in $S_{r}$ with $r \geqq \max \left\{r_{0}, r_{1}\right\}, \alpha<1$. Hence by Theorem A, $T$ has a fixed point in $\left(B_{r}-S_{r}\right)$, i.e., equation (5.5) is solvable.

THeOREM 5.4. Let $B$ be a positive symmetric mapping of $H$ into $H, \lambda>0$ and $F a$ nonlinear mapping of $H$ into $H$ such that

$(\lambda F y, y) \leqq \lambda a_{1}(y, y)+a_{2}(y, y)^{\eta} \quad\left(a_{2}>0,0<\eta<1\right) \quad$ and $\lambda a_{1}\|B\|<1$ if $a_{1}>0$.

If $A F A$ is a P-compact operator with $A^{2}=B$, then the equation $x=\lambda B F x$ is solvable.

Proof. Since $A$ is symmetric, for every $x$ in $H$,

$$
(\lambda A F A x, x)=(\lambda F A x, A x) \leqq \lambda a_{1}\|B\|\|x\|^{2}+a_{2}\|B\|^{\eta}\|x\|^{2 \eta} .
$$

It is easy to see that, since $\lambda a_{1}\|B\|<1$ if $a_{1}>0$ and $1-\eta>0$, there exists a sphere $S_{r}$ such that $\lambda a_{1}\|B\| r^{2}+a_{2}\|B\|^{n} r^{2 \eta}<r^{2}$ for $\|x\|=r$. Indeed, any fixed $r$ satisfying the inequality

$$
r>\left\{\frac{a_{2}\|B\|^{n}}{1-\lambda a_{1}\|B\|}\right\}^{1 /(2-2 n)}
$$

has this property. Hence, for any fixed $r$ satisfying (5.6),

$$
(\lambda A F A x, x) \leqq\|x\|^{2} \text { for all } x \text { in } S_{r} .
$$

Thus, as was noted in [24], the mapping $A F A$ satisfies conditions $(\Lambda)$ and $\left(\pi_{1}^{<}\right)$ on $S_{r}$. Since $\lambda>0$ and $A F A$ is $P$-compact, it follows that $\lambda A F A$ is $P$-compact. Hence, by Theorem $B$, there exists $x \in\left(B_{r}-S_{r}\right)$ such that $x=\lambda A F A x$ or $z=\lambda B F z$ with $z=A x$.

Remark 5.2. In virtue of Theorem 3 in [24] and Corollary 2.1, $A F A$ is a $P$ compact mapping of $H$ into $H$ if, for example, $F$ is a mapping of $H$ into $H$ which is either a continuous or a weakly continuous monotone decreasing mapping, a bounded demicontinuous monotone decreasing mapping or a completely continuous mapping. For the last class of mappings with $\lambda=1$, Theorem 5.4 was proved in [17].

Our next theorem contains conditions which should prove convenient and useful for applications.

THEOREM 5.5. Suppose that $A$ is a P-compact mapping of $D(A)$ into $X$ having the following properties:

(1) There exists $x_{0} \in D(A)$ and numbers $r>0$ and $c>0$ so that $B_{r}\left(x_{0}\right) \subset D(A)$ and

$$
\left\|A y-x_{0}\right\| \leqq c \text { for } y \in S_{r}\left(x_{0}\right)=\partial B_{r}(0) .
$$

(2) If $r<c$, then in addition there exists a number $q<1$ so that for $r_{1}=(c-q r) /(1-q)$, $B_{r_{1}}\left(x_{0}\right) \subset D(A)$ and $\|A x-A y\| \leqq q\|x-y\|$ for each $x \in S_{r_{1}}(0)$ and $y=\alpha x_{0}+(1-\alpha) x$ $\in S_{r}\left(x_{0}\right)$ for some $\alpha \geqq 0$.

Then $A$ has a fixed point in $B_{r^{*}}\left(x_{0}\right)$, where $r^{*}=r$ if $c \leqq r$ and $r^{*}=r_{1}$ if $c>r$. 
Proof. If $c \leqq r$, then $A\left(S_{r}\left(x_{0}\right)\right) \subset B_{r}\left(x_{0}\right)$ and, therefore, our conclusion follows from part (1) of Corollary 4.2.

Suppose now that $c>r$ and $x$ is any given element in $S_{r_{1}}\left(x_{0}\right)$, where clearly $r<c<r_{1}$. Then setting $y=\alpha x_{0}+(1-\alpha) x$ we see that $y \in S_{r}\left(x_{0}\right)$ for $\alpha=1-r / r_{1}$. Hence, it follows from (1) and (2) that $\left\|A x-x_{0}\right\| \leqq\|A x-A y\|+\left\|A y-x_{0}\right\| \leqq$ $q\|x-y\|+c$. Since $\|x-y\|=\alpha\left\|x_{0}-x\right\|=\left(1-r / r_{1}\right) r_{1}=r_{1}-r$, the last inequality and (2) imply that $\left\|A x-x_{0}\right\| \leqq q\left(r_{1}-r\right)+c=r_{1}$, i.e., $A\left(S_{r_{1}}\left(x_{0}\right)\right) \subset B_{r_{1}}\left(x_{0}\right)$. Again, by part (1) of Corollary 4.2, $A$ has a fixed point in $B_{r_{1}}\left(x_{0}\right)$.

REMARK 5.3. The conditions of Theorem 5.5 are certainly satisfied if in (1) we assume that $\left\|A y-x_{0}\right\| \leqq c$ for all $y$ in the ball $B_{r}\left(x_{0}\right)$ while, in case $r<c$, the additional condition (2) is assumed to hold for all $x$ and $y$ in the shell $r \leqq\left\|x-x_{0}\right\| \leqq r_{1}$. Under these stronger assumptions (1) and (2), Theorem 5.5 was proved in [11] for the case when $A$ is completely continuous. Note that our conditions (1) and (2) are not only weaker than the ball and shell conditions but are more suitable for applications and easier to verify.

We also remark that the application of Theorem 5.5 is easiest when the $P$ compact operator $A$ is such that the condition (2) with $q<1$ holds for all $x$ and $y$ outside a certain ball $B_{r}\left(x_{0}\right)$.

Theorem 5.5 can be applied to equations of the form

$$
\begin{aligned}
& A x=(I-T)^{-1} B(I-T) x \text { for } x \in D(B)=X \text { or } \\
& A_{1} x=(I-T)^{-1}(B-T) x \quad \text { for } x \in D(B)
\end{aligned}
$$

provided that $B$ is $P$-compact and $T$ is a bounded linear operator such that $(I-T)^{-1}$ exists and is a bounded linear operator defined on all of $X$ such that each subspace $X_{n}$ reduces $(I-T)^{-1}$, i.e., $P_{n}(I-T)^{-1}=(I-T)^{-1} P_{n}$ for each $n$. The last condition would be satisfied, for example, if $T$ were a completely continuous operator with a complete set of eigenelements $\left\{\phi_{i}\right\}(n=1,2, \ldots)$ and $X_{n}=\operatorname{span}\left\{\varphi_{1}, \ldots, \varphi_{n}\right\}$ for each $n$.

LEMMA 5.2. Under the above conditions on $B$ and $T$

(a) The operator $A=(I-T)^{-1} B(I-T)$ is $P$-compact.

(b) The operator $A_{1}=(I-T)^{-1}(B-T)$ is $P$-compact if $T$ is completely continuous.

Proof. It is obvious that the operators $P_{n} A$ and $P_{n} A_{1}$ are continuous in $X_{n}$ for $n$ greater than some $n_{0}$.

(a) Suppose now that $\left\{x_{n}\right\} \subset X$ is an arbitrary bounded sequence with $x_{n} \in X_{n}$ so that for some $p>0$

$$
g_{n}=P_{n} A x_{n}-p x_{n} \rightarrow g \text { as } n \rightarrow \infty .
$$

Since $(I-T)$ is continuous and $(I-T)^{-1}$ is reduced by $X_{n}$ for each $n$, it follows from (5.7) and the structure of $A$ that

$$
P_{n} B(I-T) x_{n}-p P_{n}(I-T) x_{n}=(I-T) g_{n} \rightarrow(I-T) g=h .
$$


Here we have used the fact (see [35]) that if a linear operator is reduced by a subspace, then so is its inverse if it exists. Setting $y_{n}=(I-T) x_{n}$, we have that $y_{n} \in X_{n}$, $\left\{y_{n}\right\}$ is a bounded set and $P_{n} B y_{n}-p y_{n} \rightarrow h$. This, and the $P$-compactness of $B$ imply that there exists a subsequence $\left\{y_{n_{i}}\right\}$ and an element $y$ in $X$ such that $y_{n_{i}} \rightarrow y$ and $P_{n_{i}} B y_{n_{i}} \rightarrow B y$. Since $(I-T)^{-1}$ is continuous, $x_{n_{i}}=(I-T)^{-1} y_{n_{i}} \rightarrow(I-T)^{-1} y=x$. Furthermore,

$$
\begin{aligned}
P_{n_{i}} A x_{n_{i}}= & P_{n_{i}}(I-T)^{-1} B(I-T) x_{n_{i}}=(I-T)^{-1} P_{n_{i}} B y_{n_{i}} \\
& \rightarrow(I-T)^{-1} B y=(I-T)^{-1} B(I-T) x=A x .
\end{aligned}
$$

Thus, $A$ is $P$-compact.

(b) With $\left\{x_{n}\right\}, p>0$ as above, suppose $T$ is completely continuous and

$$
P_{n} A_{1} x_{n}-p x_{n}=g_{n} \rightarrow g \text {. }
$$

Then, as in part (a)

$$
\begin{aligned}
P_{n}(B-T) x_{n}-p(I-T) x_{n} & =P_{n} B x_{n}-p x_{n}-(1-p) T x_{n}=P_{n}(B-(1-p) T) x_{n}-p x_{n} \\
& =(I-T) g_{n} \rightarrow(I-T) g \quad \text { as } n \rightarrow \infty .
\end{aligned}
$$

Since $(B-(1-p) T)$ is $P$-compact, there exists a subsequence $\left\{x_{n_{i}}\right\}$ and an element $x$ in $D(B)$ such that $x_{n_{i}} \rightarrow x$ and $P_{n_{i}}(B-(1-p) T) x_{n_{i}} \rightarrow(B-(1-p) T) x$. This implies that $P_{n_{i}} A_{1} x_{n_{i}} \rightarrow A_{1} x$ as $n_{i} \rightarrow \infty$, i.e., $A_{1}$ is $P$-compact.

Theorem 5.6. Let $B$ be a $P$-compact mapping of $D(B) \subset X$ into $X$. Then $B$ has at least one fixed point in $D(B)$ if the conditions of Theorem 5.5 are satisfied for

(a) The operator $A=(I-T)^{-1} B(I-T)$, where $T$ is a bounded linear mapping of $X$ into $X$ such that $(I-T)$ is continuously invertible and $X_{n}$ reduces $(I-T)^{-1}$ for each $n$.

(b) The operator $A_{1}=(I-T)^{-1}(B-T)$, where $T$ is a completely continuous operator satisfying the conditions of (a).

Proof. (a) It follows from Lemma 5.2 and our conditions that $A=(I-T)^{-1}$ $\times B(I-T)$ is $P$-compact and satisfies the conditions of Theorem 5.5. Hence there exists a ball $B_{r^{*}}\left(x_{0}\right) \subset D(B)=D(A)=X$ such that

$$
A x=(I-T)^{-1} B(I-T) x=x
$$

for some $x$ in $B_{r^{*}}\left(x_{0}\right)$. This implies that $B z=z$ for $z=(I-T) x$.

(b) As in part (a), there exists a ball $B_{\vec{r}}\left(x_{0}\right) \subset D(B)=D(A)$ such that

$$
A_{1} y=(I-T)^{-1}(B-T) y=y
$$

for some $y$ in $B_{\bar{r}}\left(x_{0}\right)$. This implies that $B y=y$.

REMARK 5.4. In case $B$ is completely continuous and $T$ is also a linear completely continuous operator, Theorem $5.6(\mathrm{~b})$ for the stronger conditions (a) and (b) was established in [11]. 
REMARK 5.5. It is not hard to see that (with $k=\left\|(I-T)^{-1}\right\|$ ) for the operator $A_{1}=(I-T)^{-1}(B-T)$, the conditions (a) and (b) of Theorem 5.5 are implied by either of the following two conditions:

(i) There are $c_{1}>0,0<m_{1}<k^{-1}$ and $r>0$ such that

$$
\left\|(B-T) x-(I-T) x_{0}\right\| \leqq c_{1} \text { for } x \text { in } S_{r}\left(x_{0}\right) \quad \text { (or for } x \text { in } B_{r}\left(x_{0}\right) \subset D(B) \text { ). }
$$

If $r<c_{1} k$ then assume also $\|(B-T) x-(B-T) y\| \leqq m_{1}\|x-y\|$ for each $x$ in $S_{r_{2}}\left(x_{0}\right)$ with $r_{2}=k\left(c_{1}-m_{1} k\right) /\left(1-m_{1} k\right)$ and $y=\alpha x_{0}+(1-\alpha) x \in S_{r}\left(x_{0}\right)$.

(ii) There are $r>0$ and $m<k^{-1}$ such that

$$
\|(B-T) x-(B-T) y\| \leqq m\|x-y\| \quad \text { if }\|x\|>r \text { and }\|y\|>r .
$$

Similarly for $A=(I-T)^{-1} B(I-T)$ a sufficient condition is either of the following conditions:

(j) There are $c>0,0<m<k^{-1}$ and $r>0$ such that

$$
\left\|B(I-T) x-(I-T) x_{0}\right\| \leqq c \text { for } x \text { in } S_{r}\left(x_{0}\right) .
$$

If $c k>r$ then assume also $\|B(I-T) x-B(I-T) y\| \leqq m\|x-y\|$ for each $x$ in $S_{r_{3}}\left(x_{0}\right)$ with $r_{3}=k(c-m k) /(1-m k)$ and $y=\alpha x_{0}+(1-\alpha) x \in S_{r}\left(x_{0}\right)$.

(jj) There are $r>0$ and $m<k^{-1}$ such that

$$
\|B(I-T) x-B(I-T) y\| \leqq m\|x-y\| \quad \text { if }\|x\|>r \text { and }\|y\|>r .
$$

We complete this section with another application of the $P$-compactness concept to the fixed point theorem for the mappings of the form $A=S+T$, where $S$ is a nonexpansive mapping and $T$ is a strongly continuous mapping of a uniformly convex Banach space $X$ into $X$. The fixed point theory for such mappings, as well as for somewhat more general (e.g., semicontractive) mappings, has been recently developed by Browder [4] who in his discussion employed the entire machinery of monotone and $J$-monotone operators. In our discussion below we will not use the theory of $J$-monotone operators defined below. We should add that, as was shown in [4], $A=S+T$ need not have a fixed point if we assume that $T$ is only completely continuous even if $A$ maps $B_{r}(0) \subset H$ into $B_{r}(0)$. Furthermore, it is known that if $X$ is a general Banach space, then a nonexpansive mapping $A$ of $B_{r}(0)$ into $B_{r}(0)$ need not have a fixed point. Thus, certain restrictions on $A$ and/or $X$ have to be imposed in order for $A$ to have fixed points.

Let $\mu(r)$ be a continuous strictly increasing real-valued function with $\mu(0)=0$. A mapping $J$ of $X$ into $X^{*}$ is called a duality mapping with gauge function $\mu$ if $\|J x\|=\mu(\|x\|)$ and $(J x, x)=\|x\| \mu(\|x\|)$ for each $x$ in $X$. An operator $A$ of $X$ into $X$ is said to be $J$-monotone if $(A x-A y, J(x-y)) \geqq 0$ for all $x$ and $y$ in $X$. For the discussion of duality mappings and $J$-monotone operators see [4], [5].

In what follows we shall make use of the following two lemmas which in a somewhat different form were first proved in [22]. For the sake of completeness we prove here these lemmas by using a combination of arguments in [33], [22], [28]. 
LEMMA 5.3. Let $X$ be a uniformly convex Banach space having a weakly continuous duality mapping $J$ of $X$ into $X^{*}$. Let $\left\{x_{n}\right\}$ be a sequence in $X$ such that $x_{n} \rightarrow x_{0}$. Then there exists a subsequence $\left\{x_{m}\right\}$ of $\left\{x_{n}\right\}$ such that for any $x$ in $X$

$$
\lim _{m}\left\|x_{m}-x\right\| \geqq \lim \left\|x_{m}-x_{0}\right\|
$$

with equality holding in (5.9) if and only if $x=x_{0}$.

Proof. It is obvious that there exists a subsequence $\left\{x_{m}\right\}$ of $\left\{x_{n}\right\}$ such that $\left\|x_{m}-x_{0}\right\| \rightarrow d_{0}$ and $\left\|x_{m}-x\right\| \rightarrow d$ as $m \rightarrow \infty$. Hence, since $x_{m}-x_{0} \rightarrow 0$ implies $J\left(x_{m}-x_{0}\right) \rightarrow 0$, the definition of $J$ and the passage to the limit in

$$
\left(J\left(x_{m}-x_{0}\right), x_{m}-x_{0}\right) \leqq\left\|J\left(x_{m}-x_{0}\right)\right\|\left\|x_{m}-x\right\|+\left|\left(J\left(x_{m}-x_{0}\right), x-x_{0}\right)\right|
$$

imply that $\mu\left(d_{0}\right) d_{0} \leqq \mu\left(d_{0}\right) d$ from which (5.9) follows. To complete the proof, suppose that $d_{0}=d$ in (5.9). Since

$$
\left\|x_{m}-\left(t x+(1-t) x_{0}\right)\right\| \leqq t\left\|x_{m}-x\right\|+(1-t)\left\|x_{m}-x_{0}\right\|
$$

for any $t$ in [0,1], it follows from the first part of Lemma 5.3 and our assumption $d_{0}=d$ that $\left\|x_{m}-x_{0}\right\| \rightarrow d_{0},\left\|x_{m}-x\right\| \rightarrow d_{0}$, and $\left\|x_{m}-\left(t x+(1-t) x_{0}\right)\right\| \rightarrow d_{0}$. Hence, by uniform convexity of $X,\left\|\left(x_{m}-x_{0}\right)-\left(x_{m}-x\right)\right\| \rightarrow 0$, i.e., $x=x_{0}$.

A consequence of Lemma 5.3 is the following useful result.

LEMMA 5.4. Let $X$ be a uniformly convex Banach space with a weakly continuous duality mapping $J$ of $X$ into $X^{*}$. If $A=S+T$ maps a closed convex set $D(A)=C$ of $X$ into $X$, where $S$ is nonexpansive on $C$ and $T$ strongly continuous, then $(I-A)$ is strongly closed.

Proof. Let $\left\{x_{n}\right\}$ be a sequence in $C$ so that $x_{n} \rightarrow x_{0}$ and let $x_{n}-A x_{n} \rightarrow y_{0}$ for some $y_{0}$ in $X$. Since $C$ is closed and convex and hence weakly closed, $x_{0}$ lies in $C$ and $T x_{n} \rightarrow T x_{0}$ by strong continuity of $T$. Hence it suffices to show that $(I-S) x_{0}$ $=y_{0}+T x_{0}$. Let $\left\{x_{m}\right\}$ be a subsequence of $\left\{x_{n}\right\}$ so that $\lim _{m}\left\|x_{m}-x_{0}\right\|$ exists. Since $(I-S) x_{m} \rightarrow y_{0}+T x_{0}$, there exists a sequence $z_{m} \rightarrow 0$ such that $S x_{m}=x_{m}-y_{0}$ $-T x_{0}+z_{m}$ and

$$
\begin{aligned}
\left\|x_{m}-x_{0}\right\| & \geqq\left\|S x_{m}-S x_{0}\right\|=\left\|x_{m}-y_{0}-T x_{0}-S x_{0}+z_{m}\right\| \\
& \geqq\left\|x_{m}-y_{0}-T x_{0}-S x_{0}\right\|-\left\|z_{m}\right\|,
\end{aligned}
$$

whence, since $z_{m} \rightarrow 0$, we deduce

$$
\lim _{m}\left\|x_{m}-x_{0}\right\| \geqq \lim _{m}\left\|x_{m}-y_{0}-T x_{0}-S x_{0}\right\| .
$$

It follows from this and Lemma 5.3 that $x_{0}=y_{0}-T x_{0}-S x_{0}=y_{0}-A x_{0}$.

REMARK 5.6. Lemma 5.4 was first proved (for $T=0$ ) in a more general setting in [4] using the theory of $J$-monotone operators under the assumption that $A$ is defined on all of $X$. In a case of a Hilbert space a somewhat different proof is given in [7]. 
THEOREM 5.7. Let $X$ be a uniformly convex Banach space with a weakly continuous duality mapping $J$ of $X$ into $X^{*}$ and with $\left\|P_{n}\right\|=1$ for all $n$. Let $A=S+T$ be a mapping of $X$ into $X$, where $S$ is nonexpansive and $T$ is strongly continuous. Suppose that for some bounded closed convex set $C$ with 0 in int $C, A$ satisfies $\left(\pi_{1}^{<)}\right.$on $\partial C$. Then there exists a point $x_{0}$ in $(C-\partial C)$ such that $S x_{0}+T x_{0}=x_{0}$.

Proof. By Corollary 2.4, $S-I$ is $P$-compact; furthermore, since a uniformly convex Banach space is reflexive and $T$ is strongly continuous, it follows that $T$ is completely continuous and, therefore, by Lemma $2.1, S+T-I$ is $P$-compact. Suppose now that $(A-I) x=\alpha x$ for some $x$ in $\partial C$. Then $A x=(1+\alpha) x$ and therefore, since $A$ satisfies condition $\left(\pi_{1}^{<}\right)$on $\partial C, \alpha \leqq 0$. Hence, by Theorem 4.2 , for each $\mu_{k}>0$ with $\mu_{k} \rightarrow 0$ as $k \rightarrow \infty$ there exists an element $x_{k}$ in $(C-\partial C)$ such that $(A-I) x_{k}=\mu_{k} x_{k}$. But, since $\left\{x_{n}\right\} \subset C$, a closed bounded convex set, and $X$ is reflexive, passing to a subsequence we may assume that $x_{k} \rightarrow x_{0}$, where $x_{0}$ is some element in $C$. Thus $x_{k} \rightarrow x_{0}$ and $(I-A) x_{k}=-\mu_{k} x_{k} \rightarrow 0$ as $k \rightarrow \infty$. Since, by Lemma 5.4, $(I-A)$ is strongly closed, it follows that $(I-A) x_{0}=0$ or $S x_{0}+T x_{0}=x_{0}$.

REMARK 5.7. As was already noted, Theorem 5.7 was proved in [4] by means of the theory of $J$-monotone operators for the class of semicontractive mappings $U$ of $X$ into $X$ (which contains mappings $A=S+T$ ) but under the stronger condition, namely, that $U$ maps $C$ into $C$ which, for $U=\mathrm{S}+T$ reduces to the requirement that $S x+T y \in C$ for all $x$ and $y$ in $C$.

\section{Iterative and general approximation methods for $P$-compact mappings.}

ITERATIVE METHOD. Extending certain results of [16], [33] and [26], among other results, it was shown in [6] that if $X$ is a uniformly convex Banach space, $C$ a closed convex subset of $X$ and $A$ a contractive (nonexpansive) mapping of $C$ into $C$ with a nonempty set of fixed points in $C$, then for each given $x_{0}$ in $C$ and each fixed $\lambda$ in $(0,1)$, the sequence $\left\{x_{n+1}\right\}$ determined by the iteration method

$$
x_{n+1}=\lambda A x_{n}+(1-\lambda) x_{n} \quad(n=0,1,2, \ldots)
$$

converges strongly to a fixed point of $A$ in $C$ provided $A$ satisfies the condition

$$
(I-A) \text { maps every bounded closed subset of } C \text { into a closed subset. }
$$

It is easy to see that condition $(\alpha)$ is implied by the stronger assumption that $A$ is completely continuous. Furthermore, condition $(\alpha)$ is a slight generalization of the concept of demicompactness $\left({ }^{6}\right)$ of $A$ introduced and studied in [26].

$\left.{ }^{(}\right) A$ is said to be demicompact if whenever $\left\{u_{n}\right\}$ is a bounded sequence and $\left\{u_{n}-A u_{n}\right\}$ is strongly convergent, then $\left\{u_{n}\right\}$ contains a strongly convergent subsequence. It was asserted in [6] that condition $(\alpha)$ is equivalent to the demicompactness. This is clearly not the case for, as was observed in [28], $A=I$ satisfies condition $(\alpha)$ but is not demicompact. The authors of [6] caught this inaccuracy too late for the correction to be inserted in [6]. For details, historical development and recent contributions to the iterative method for the construction of fixed points of contractive type mappings see [7], [28]. 
To discuss the problem of convergence of the method (6.1) in case $A$ is a $P$ compact mapping we first derive some preliminary results.

LemMa 6.1. (a) Let $A$ be a nonexpansive $P_{1}$-compact mapping of $D(A)=X$ into $X$. Then A satisfies condition ( $\alpha$ ).

(b) Suppose additionally that $\left\|P_{n}\right\|=1$ for all $n$. Then if $A$ is a nonexpansive $P_{1}$ compact mapping of $D(A)=B_{r}(0)$ into $X$, then $A$ satisfies condition $(\alpha)$ on $B_{r}(0)$ (i.e., $(I-A)$ maps every closed subset of $B_{r}(0)$ into a closed subset of $\left.X\right)$.

Proof. Let $Q$ be a bounded closed set in $X$ and let $\left\{u_{k}\right\}$ be a sequence in $Q$ such that $(I-A) u_{k} \rightarrow v$ as $k \rightarrow \infty$, where $v$ is some element in $X$. Since the system $\left(\left\{X_{n}\right\},\left\{P_{n}\right\}\right)$ is projectionally complete in $X$, for each integer $k>0$ there exists an integer $n(k)>k$ such that $\left\|u_{k}-w_{n(k)}\right\|<1 / k$, where we have put $w_{n(k)}=P_{n(k)} u_{k}$. This and the relation $(I-A) u_{k} \rightarrow v$ as $k \rightarrow \infty$ imply that

$$
\begin{aligned}
\left\|P_{n(k)} A w_{n(k)}-w_{n(k)}+v\right\| \leqq & \left\|P_{n(k)} A w_{n(k)}-P_{n(k)} A u_{k}\right\| \\
& +\left\|P_{n(k)} A u_{k}-w_{n(k)}+P_{n(k)} v\right\|+\left\|v-P_{n(k)} v\right\| .
\end{aligned}
$$

Since $\left\{P_{n}\right\}$ is uniformly bounded by $K \geqq 1, A$ is nonexpansive, and $w_{n(k)}=P_{n(k)} u_{k}$, it follows from the above inequality and our assumptions that

$$
\begin{aligned}
\left\|P_{n(k)} A w_{(k)}-w_{n(k)}+v\right\| \leqq & K\left\|w_{n(k)}-u_{k}\right\|+K\left\|A u_{k}-u_{k}+v\right\| \\
& +\left\|v-P_{n(k)} v\right\| \rightarrow 0 \text { as } k \rightarrow \infty
\end{aligned}
$$

Since $\left\{w_{n(k)} \mid w_{n(k)} \in X_{n(k)}\right\}$ is bounded and $A$ is $P_{1}$-compact, the preceding relation implies that there exists a subsequence $\left\{w_{n(j)}\right\}$ of $\left\{w_{n(k)}\right\}$ and an element $w$ such that $w_{n(j)} \rightarrow w$ and $P_{n(j)} A w_{n(j)} \rightarrow A w$ as $j \rightarrow \infty$. Consequently, $\left\|u_{j}-w\right\| \leqq\left\|u_{j}-w_{n(j)}\right\|$ $+\left\|w_{n(j)}-u\right\| \rightarrow 0$ as $j \rightarrow \infty$. Since $\left\{u_{j}\right\}$, being a subsequence of $\left\{u_{k}\right\}$, lies in $Q$ and $Q$ is closed it follows that $w \in Q$ and $A w-w=v$, i.e., $(I-A)$ satisfies condition $(\alpha)$.

The validity of assertion (b) follows from the same arguments as above since the condition that $Q \subset B_{r}(0)$ and that $\left\|P_{n}\right\|=1$ for all $n$ imply that $w_{n(k)} \in B_{r} \cap X_{n(k)}$ for all $n(k)$. The rest of the proof is the same as that of (a).

LEMMA 6.2. Let $A$ be a continuous $P_{1}$-compact mapping of a bounded closed convex set $C$ with interior into $X$ and let $F$ be a closed convex set lying in Int $C$ such that $A x \neq x$ for all $x$ in $(C-F)$. If we denote by $R(F, \alpha)=\{x \in C \mid d(x, F) \geqq \alpha\}$ for some $\alpha>0$, then for each given $\alpha>0$ there exists an $\varepsilon=\varepsilon(\alpha)>0$ such that $\|x-A x\|>\varepsilon$ for all $x$ in $R(F, \alpha)$.

Proof. Let us remark that in view of our conditions on $F$ the set $R(F, \alpha)$ is not empty for sufficiently small $\alpha>0$. First we show that to a given $\alpha>0$ there exists an $\bar{\varepsilon}(\alpha)>0$ and an integer $n_{0}>0$ such that for all $n \geqq n_{0}$

$$
\left\|x-P_{n} A x\right\|>\bar{\varepsilon}(\alpha) \text { for all } x \text { and } y \text { in } X_{n} \cap R(F, \alpha) \text {. }
$$

If this were not the case, then there would exist a number $\alpha_{0}>0$ and a sequence $\left\{x_{m} \mid x_{m} \in X_{m} \cap C\right\}$ such that $d\left(x_{m}, F\right) \geqq \alpha_{0}$ and

$$
\left\|x_{m}-P_{m} A x_{m}\right\| \rightarrow 0 \text { as } m \rightarrow \infty \text {. }
$$


Thus, by $P_{1}$-compactness of $A$, there exists a subsequence $\left\{x_{m_{i}}\right\}$ and an element $x$ in $C$ such that $x_{m_{i}} \rightarrow x$ and $P_{m_{i}} A x_{m_{i}} \rightarrow A x$, as $m_{i} \rightarrow \infty$, and $x-A x=0$ contradicting the fact that $d\left(x_{m_{i}}, F\right) \geqq \alpha_{0}$ and $x_{m_{t}} \rightarrow x$. Hence, (6.2) holds. Furthermore, since $P_{m} x=x$ for $x$ in $X_{m}$ and $\left\|P_{n}\right\| \leqq K$, it follows from (6.2) that

$$
\|x-A x\| \geqq \varepsilon(\alpha)=\bar{\varepsilon}(\alpha) / K \text { for all } x \text { in } X_{n} \cap R(F, \alpha) \text { and all } n \geqq n_{0} .
$$

Note that (6.2) and (6.3) have been derived without using the continuity of $A$.

Now let $y$ be an arbitrary element in $R(F, \alpha)$. There are four possible cases: (a) $d(y, F)>\alpha$ and $y \notin \partial C$; (b) $d(y, F)>\alpha$ and $y \in \partial C$; (c) $d(y, F)=\alpha$ and $y \notin \partial C$; (d) $d(y, F)=\alpha$ and $y \in \partial C$.

(a) Since $d(y, F)>\alpha$ and $y \notin \partial C$, there exists a ball $B_{\delta}(y)$ about $y$ with radius $\delta>0$ such that $B_{\delta}(y) \subset C \cap R(F, \alpha)$. Since $\bigcup_{n}^{\infty} X_{n}$ is dense in $X$, we can find a sequence $x_{n} \in X_{n}$ such that $x_{n} \rightarrow y$ as $n \rightarrow \infty$. Hence, there exists an $N>0$ such that $x_{n} \in B_{\delta}(y)$ for all $n \geqq N$. Consequently, (6.3) implies that $\left\|x_{n}-A x_{n}\right\|>\varepsilon(\alpha)$ for all $n \geqq N$. This and the continuity of $A$ implies that $\|y-A x\| \geqq \varepsilon(\alpha)$.

Cases (b), (c), and (d) can be handled by one technique. Suppose for definiteness that $d(y, F)=\alpha$ and $y \notin \partial C$. In virtue of our conditions on $F$, we can find a sequence $\left\{y_{n}\right\}$ in $R(F, \alpha)$ such that $d\left(y_{n}, F\right)=\alpha_{n}>\alpha$ and $y_{n} \rightarrow y$ as $n \rightarrow \infty$. Then for each $n$, by case (a), $\left\|y_{n}-A y_{n}\right\|>\varepsilon(\alpha)$ and hence again, by continuity of $A$, we have $\|y-A y\|$ $\geqq \varepsilon(\alpha)$ for all $y$ in $R(F, \alpha)$.

REMARK 6.1. An analogous lemma for completely continuous operators is known (e.g., see [17]) to play a very important role in the study of completely continuous operator equations. It is, therefore, expected that Lemma 6.2 will also be of considerable value in the study of $P$-compact operator equations. Its importance in our investigation will be seen below.

THEOREM 6.1. Let $X$ be a uniformly convex Banach space and $A$ a nonexpansive $P_{1}$-compact mapping of $D(A) \subset X$ into $X$.

(a) If $D(A)=X$ and, for some bounded closed convex set $C$ with an interior, $A$ maps $C$ into $C$. Then for any $x_{0} \in C$ and any fixed $\lambda$ in $(0,1)$ the sequence $x_{n+1}$ determined by the iteration method (6.1) converges strongly to a fixed point of $A$ in $C$.

(b) If $D(A)=B_{r}(0)$ and $A$ maps $B_{r}(0)$ into $B_{r}(0)$, then the same assertion holds provided $\left\|P_{n}\right\|=1$.

(c) If $D(A)=C$, where $C$ is as in (a), and $A$ is a map of $C$ into $C$ with no fixed points on $\partial C$, then the assertion of (a) remains valid.

Proof. (a) In virtue of Lemma 6.1(a) and the result in [6] quoted above, it suffices to prove that $A$ has a fixed point in $C$. But, under our conditions on $A$ and $C$, Corollary 4.2 implies that $A$ has fixed points in $C$.

(b) The proof of Theorem 6.1(b) follows from the same arguments and Lemma 6.1(b).

(c) To prove assertion (c) first note that, by Corollary 4.2, the set $F$ of fixed points of $A$ in $C$ is nonempty and, in fact, $F$ is a convex closed set (see [33]). Since, 
by assumption, $A$ has no fixed points on the boundary $\partial C, F \subset \operatorname{Int} C$. Now, it is easy to show (see [16]) that, for the sequence $\left\{x_{n+1}\right\}$ defined by $(6.1), d\left(x_{n+1}, F\right)$ $\leqq d\left(x_{n}, F\right)$ for each $n$. Hence, $\left\{d\left(x_{n}, F\right)\right\}$ is a monotonically decreasing sequence of real numbers which is bounded below by zero. We claim that $d\left(x_{n}, F\right) \rightarrow 0$ as $n \rightarrow \infty$. Suppose, to the contrary, that $d\left(x_{n}, F\right) \rightarrow \alpha_{0}>0$ as $n \rightarrow \infty$. By monotonicity, $d\left(x_{n}, F\right)>\alpha_{0}$ for each $n$. Hence, by Lemma 6.2, there exists $\varepsilon_{0}=\varepsilon\left(\alpha_{0}\right)>0$ such that $\left\|x_{n}-A x_{n}\right\| \geqq \varepsilon_{0}$. Letting $v_{n}=x_{n}-u$ and $w_{n}=A x_{n}-u$ with $u$ in $F$, we see that, since $\left\|x_{n}-A x_{n}\right\| \leqq\left\|x_{n-1}-A x_{n-1}\right\|,\left\|w_{n}-v_{n}\right\|>\varepsilon_{0}$ for $n \geqq 0$ and

$$
\left\|x_{n+1}-u\right\|=\left\|\lambda\left(A x_{n}-A u\right)+(1-\lambda)\left(x_{n}-u\right)\right\|=\left\|\lambda w_{n}+(1-\lambda) v_{n}\right\| \text {. }
$$

Hence, by Theorem 1 in [33], there exists a $\delta_{0}=\delta\left(\varepsilon_{0}, \lambda, C\right)$ so that

$$
\left\|x_{n+1}-u\right\|<\left(1-\delta_{0}\right)\left\|x_{n}-u\right\| \text { for each } n \text {. }
$$

Consequently, $\left\|x_{n}-A x_{n}\right\|<2\left(1-\delta_{0}\right)\left\|x_{n-1}-u\right\|$. The last two relations imply that $\left\|x_{n}-A x_{n}\right\| \rightarrow 0$ as $n \rightarrow \infty$. Hence, the contradiction is reached and, therefore, $d\left(x_{n}, F\right) \rightarrow 0$ as $n \rightarrow \infty$. From the arguments of Krasnoselsky [16] it then follows that $\left\{x_{n+1}\right\}$ is a Cauchy sequence. Since $C$ is closed and $\left\{x_{n+1}\right\} \in C$, there exists an $x$ in $C$ such that $x_{n} \rightarrow x$ as $n \rightarrow \infty$. The continuity of $A$ then implies that $x$ is a fixed point of $A$.

Note. We note, for example, that if $X$ is such that $\left\|P_{n}\right\|=1$ then Theorem 6.1(a) is applicable to nonexpansive operators of the form $A=S+T$, where $S$ is Lipschitzian on $D(A)=X$ with $L<1$ and $T$ is completely continuous; if, however, $A=S+T$ is defined only on $D(A)=B_{r}(0)$ and $L<1 / 2$, then Theorem $6.1(\mathrm{~b})$ is applicable. Note that in both cases $A$ is $P_{1}$-compact but not completely continuous so that the results in [16], [33] are not applicable.

ApProximation methods. Let $A$ be a bounded continuous $P$-compact mapping of $D(A)=X$ into $X$. In this section we extend certain results of [24] concerning the projection method for the approximate solution of the equation

$$
A x=x
$$

to the approximations of a more general type. Our results will in particular include the recent results of Vainikko [39] for completely continuous mappings.

For the approximate solution of equation (6.4) we take the solutions of the equations

$$
A_{n} x=x
$$

where the $A_{n}$ are bounded $P$-compact mappings of $X$ into $X$ which in some sense (to be defined below) approximate $A$.

TheOrem 6.2. Let $A$ and $A_{n}$ be as above. Suppose that $A$ satisfies condition $\left(\pi_{1}^{<}\right)$ on $\partial B_{r}$, for some $r>0$. Let $A$ have at most one fixed point in $\left(B_{r}-\partial B_{r}\right)$. Suppose further that

$$
\tau_{n}=\sup _{x \in B_{r}}\left\|A_{n} x-A x\right\| \rightarrow 0 \text { as } n \rightarrow \infty
$$


Then $A$ has a (unique) fixed point $x_{0}$ in $\left(B_{r}-\partial B_{r}\right)$ and there exists an integer $N>0$ such that for each $n \geqq N$ equation (6.5) has a solution $x_{n}$ in $\left(B_{r}-\partial B_{r}\right)$ so that $x_{n} \rightarrow x_{0}$ as $n \rightarrow \infty$.

If, in addition, we assume that $A_{n}(x)$ are uniformly in $n$ Fréchet-differentiable at $x=x_{0}$ and that, for sufficiently large $n$,

$$
\left\|x-A_{n}^{\prime}\left(x_{0}\right) x\right\| \geqq \beta\|x\| \quad \text { for each } x \text { in } B_{r}
$$

and some constant $\beta>0$, then the estimate of the error $\left\|x_{n}-x_{0}\right\|$ is given by

$$
\left\|x_{n}-x_{0}\right\| \leqq \frac{1}{\beta-\theta_{n}}\left\|A_{n} x_{0}-A x_{0}\right\|
$$

where $\theta_{n} \rightarrow 0$ as $n \rightarrow \infty$.

Proof. First note that in virtue of our conditions on $A$, Theorem $\mathrm{A}$ implies that $A$ has a fixed point $x_{0}$ in $\left(B_{r}-\partial B_{r}\right)$ which, by assumption, is unique. Now by Lemma 6.2, there exists an $\varepsilon$ such that for all $x$ in $\partial B_{r},\|x-A x\| \geqq \varepsilon$. Since $\tau_{n} \rightarrow 0$ as $n \rightarrow \infty$ we can find an $N$ such that for all $n \geqq N, \tau_{n} \leqq \varepsilon / 2$. Then, for all $x$ in $\partial B_{r}$ and all $n \geqq N$

$$
\left\|x-A_{n} x\right\| \geqq\|x-A x\|-\left\|A_{n} x-A x\right\| \geqq \varepsilon / 2 .
$$

Thus, for $n \geqq N, A_{n}$ have no fixed point on $\partial B_{r}$, and so by results of Tucker [36], for all $n \geqq N$, there exist $x_{n}$ in $\left(B_{r}-\partial B_{r}\right)$ such that $A_{n} x_{n}-x_{n}=0$.

Now let $\varepsilon>0$ be arbitrary and consider the set $F_{x}$ of all $x$ in $B_{r}$ such that $\left\|x-x_{0}\right\|$ $\geqq \varepsilon$. Since $x_{0}$ is the unique fixed point of $A$ in $B_{r}$, by Lemma 6.2, there exists $\alpha(\varepsilon)>0$ such that $\|x-A x\| \geqq \alpha(\varepsilon)$ for $x$ in $F_{x}$. As above, for sufficiently large $n$ and all $x$ in $F_{x}$, we have

$$
\left\|x-A_{n} x\right\| \geqq\|x-A x\|-\left\|A x-A_{n} x\right\| \geqq \alpha(\varepsilon)-\alpha(\varepsilon) / 2=\alpha(\varepsilon) / 2>0 .
$$

Thus, for sufficiently large $n$, the approximate equations (6.5) cannot have solutions $x_{n}$ outside an $\varepsilon$-neighborhood of $x_{0}$. Since $\varepsilon$ is arbitrary, $\left\|x_{n}-x_{0}\right\| \rightarrow 0$ as $n \rightarrow \infty$.

To prove the second part of Theorem 6.2 let us first observe that since $A_{n}^{\prime}$ is a Fréchet derivative of $A_{n}(x)$ at $x=x_{0}$ it follows that

$$
A_{n}\left(x_{n}\right)=A_{n}\left[x_{0}+\left(x_{n}-x_{0}\right)\right]=A_{n} x_{0}+A_{n}^{\prime}\left(x_{n}-x_{0}\right)+w_{n}\left(x_{0} ; x_{n}-x_{0}\right) .
$$

Furthermore, since $A_{n}(x)$ are uniformly in $n$ Fréchet-differentiable and, by the first part of our Theorem, $x_{n} \rightarrow x_{0}$ as $n \rightarrow \infty$, it follows that

$$
\theta_{n}=\frac{\left\|w_{n}\left(x_{0} ; x_{n}-x_{0}\right)\right\|}{\left\|x_{n}-x_{0}\right\|}=\frac{\left\|A_{n} x_{n}-A_{n} x_{0}-A_{n}^{\prime}\left(x_{n}-x_{0}\right)\right\|}{\left\|x_{n}-x_{0}\right\|} \rightarrow 0,
$$


as $n \rightarrow \infty$. Using the above relation and the fact that $A x_{0}=x_{0}$ and $A_{n} x_{n}=x_{n}$ we obtain

$$
\begin{aligned}
x_{n}-x_{0}=A_{n} x_{n}-A x_{0} & =A_{n} x_{n}-A_{n} x_{0}+A_{n} x_{0}-A x_{0} \\
& =A_{n}^{\prime}\left(x_{0}\right)\left(x_{n}-x_{0}\right)+w_{n}\left(x_{0} ; x_{n}-x_{0}\right)+A_{n} x_{0}-A x_{0},
\end{aligned}
$$

whence we get the looked-for equality

$$
\left\{I-A_{n}^{\prime}\left(x_{0}\right)\right\}\left(x_{n}-x_{0}\right)=A_{n} x_{0}-A x_{0}+w_{n}\left(x_{0} ; x_{n}-x_{0}\right) .
$$

In view of (6.6) and the equality (6.8), we derive from (6.9) the following

$$
\beta\left\|x_{n}-x_{0}\right\| \leqq\left\|A_{n} x_{0}-A x_{0}\right\|+\theta_{n}\left\|x_{n}-x_{0}\right\| .
$$

Since, by (6.8), $\theta_{n} \rightarrow 0$ as $n \rightarrow \infty$, we see that $\beta-\theta_{n}>0$ for sufficiently large $n$. Hence the error estimate follows from (6.10). This completes the proof of Theorem 6.2 .

Remark 6.2. In case $A_{n}=P_{n} A$, Theorem 6.2 reduces to Theorem 7 in [24] for projectional methods under somewhat weaker conditions.

REMARK 6.3. If, in Theorem 6.2 , we replace $B_{r}(0)$ by $B_{r}\left(x_{0}\right)$, then by employing a translation process similar to that used in Theorem 4.4 , we obtain the corresponding result of Vainikko [39] when $A$ and $A_{n}$ are completely continuous.

REMARK 6.4. Finally, we remark that if additionally we assume that $A_{n}(x)$ are uniformly in $n$ continuous Fréchet-differentiable at $x=x_{0}$, then by arguments analogous to those in [39] one can show that for sufficiently large $n$, the approximate equations are uniquely solvable.

It follows from Theorem 6.2 that if $x_{0}$ is a fixed point of a nonlinear mapping $A$, it is important to know under what conditions $x_{0}$ is an isolated fixed point of $A$. In this direction we prove the following theorem.

THEOREM 6.3. Let $x_{0}$ be a fixed point of a mapping $A$. If $A(x)$ has a P-compact Fréchet derivative $A^{\prime}\left(x_{0}\right)$ at $x=x_{0}$ such that $x=0$ is the only solution of the equation $x-A^{\prime}\left(x_{0}\right) x=0$, then $x_{0}$ is an isolated fixed point of $A$.

Proof. Since $A^{\prime}\left(x_{0}\right)$ is a linear $P$-compact mapping of $X$ into $X$ and $\lambda=1$ is not an eigenvalue of $A^{\prime}\left(x_{0}\right)$, it follows from Theorem 3.1 and Lemma 6.2 that there exists a constant $c>0$ such that $\left\|A^{\prime}\left(x_{0}\right) x-x\right\| \geqq c\|x\|$ for all $x$ in $X$. Since, by definition of $A^{\prime}\left(x_{0}\right)$, for each $h$ in $X, A\left(x_{0}+h\right)-A\left(x_{0}\right)=A^{\prime}\left(x_{0}\right) h+w\left(x_{0} ; h\right)$, where $A^{\prime}\left(x_{0}\right)$ is a linear mapping of $X$ into $X$ and

$$
\lim _{\|h\| \rightarrow 0} \frac{\left\|w\left(x_{0} ; h\right)\right\|}{\|h\|}=0
$$

it follows from (6.11) that there exists a number $r_{0}>0$ such that $\|x\|<r_{0}$ implies the inequality

$$
\left\|w\left(x_{0} ; x\right)\right\|=\left\|A\left(x_{0}+x\right)-A\left(x_{0}\right)-A^{\prime}\left(x_{0}\right) x\right\| \leqq \frac{c}{2}\|x\| .
$$


Thus, on the spheres $S_{r}\left(x_{0}\right)$ of radii $r \leqq r_{0}, A$ has no fixed points since

$$
\left\|A\left(x_{0}+x\right)-\left(x_{0}+x\right)\right\| \geqq\left\|A^{\prime}\left(x_{0}\right) x-x\right\|-\left\|w\left(x_{0} ; x\right)\right\| \geqq\left(c-\frac{c}{2}\right)\|x\|=\frac{c}{2}\|x\| .
$$

This shows that $x_{0}$ is an isolated fixed point of $A$.

Remark 6.5. If $X=H, x_{0}$ is a fixed point of a mapping $A$ of $D(A)$ into $H$ and $A$ has a Fréchet derivative $A^{\prime}\left(x_{0}\right)$ at $x=x_{0}$ then, for example, by Corollary 2.1 the mapping $A^{\prime}\left(x_{0}\right)$ is $P$-compact if $\left(A^{\prime}\left(x_{0}\right) h, h\right) \leqq 0$ for all $h$ in $H$. Note that $A^{\prime}\left(x_{0}\right)$ need not be symmetric.

\section{REFERENCES}

1. M. Altman, A fixed point theorem in Banach spaces, Bull. Acad. Polon. Sci. Cl. III 5 (1957), 19-22.

2. F. E. Browder, Nonlinear elliptic boundary value problems, Bull. Amer. Math. Soc. 69 (1963), 862-874.

3. - Solvability of nonlinear functional equations, Duke Math. J. 30 (1963), 557-566.

4. —_ Fixed point theorems for nonlinear semicontractive mappings in Banach spaces,

Arch. Rational Mech. Anal. 21 (1966), 259-269.

5. F. E. Browder and D. G. DeFigueiredo, J-monotone nonlinear operators in Banach spaces, Nederl. Akad. Wetensch. Proc. Ser. A. 69 (1966), 412-420.

6. F. E. Browder and W. V. Petryshyn, The solution by iteration of nonlinear functional equations in Banach spaces, Bull. Amer. Math. Soc. 72 (1966), 571-575.

7. - Construction of fixed points of nonlinear mappings in Hilbert space, J. Math. Anal. Appl. 20 (1967), 197-228.

8. D. G. DeFigueiredo, Fixed point theorems for nonlinear operators and Galerkin approximations, J. Differential Equations 3 (1967), 271-281.

9. - Some remarks on fixed point theorems for nonlinear operators in Banach space (to appear).

10. C. L. Dolph and G. J. Minty, "On nonlinear integral equations of the Hammerstein type" in Nonlinear integral equations, edited by Anselone, Univ. of Wisconsin Press, Madison, Wis., 1967.

11. H. H. Ehrmann, On implicit function theorems and the existence of solutions of nonlinear equations, Enseignement Math. (3) 9 (1963), 129-176.

12. H. Hanani, E. Netanyaku and M. Reichaw-Reichbach, The sphere in the image, Israel J. Math. 1 (1963), 188-195.

13. R. I. Kacurowsky, On monotone operators and convex functionals, Uspehi Mat. Nauk 15 (1960), 213-215.

14. S. Kaniel, Quaiscompact nonlinear operators in Banach space and applications, Arch. Rational Mech. Anal. 20 (1965), 259-278.

15. T. Kato, Demicontinuity, hemicontinuity, and monotonicity, Bull. Amer. Math. Soc. 70 (1964), 548-550.

16. M. A. Krasnoselsky, Two remarks on the method of successive approximations, Uspehi Mat. Nauk 10 (1955), no. 1 (63), 123-127.

17. — Topological methods in the theory of nonlinear integral equations, Macmillan, New York, 1964.

18. M. Lees and M. H. Schultz, A Leray-Schauder principle for A-compact mappings and the numerical solution of nonlinear two-point boundary value problems, Wiley, New York, 167-179. 
19. J. Leray, La théorie des pointes fixés et ses applications en analyse, Proc. Internat. Congress Math., 1950, vol. 2, pp. 202-208, Amer. Math. Soc., Providence, R. I., 1952.

20. J. Massera and J. J. Schaffer, Linear differential equations and functional analysis. I, Ann. of Math. 67 (1958), 517-573.

21. G. J. Minty, Monotone (nonlinear) operators in Hilbert space, Duke Math. J. 29 (1962), 341-346.

22. Z. Opial, Weak convergence of the sequence of successive approximations for nonexpansive mappings, Bull. Amer. Math. Soc. 73 (1967), 591-597.

23. W. V. Petryshyn, On a fixed point theorem for nonlinear P-compact operators in Banach space, Bull. Amer. Math. Soc. 72 (1966), 329-334.

24. - On nonlinear P-compact operators in Banach space with application to constructive fixed point theorems, J. Math. Anal. Appl. 15 (1966), 228-242.

25. - Further remarks on nonlinear P-compact operators in Banach space, Proc. Nat. Acad. Sci. U.S.A. 55 (1966), 684-687 (extended version in J. Math. Anal. Appl. 16 (1966), 243-253).

26. - Construction of fixed points of demicompact mappings in Hilbert space, J. Math. Anal. Appl. 14 (1966), 276-284.

27. - On the extension and the solution of non-linear operator equations, Illinois J. Math. 10 (1966), 255-274.

28. _ Iterative construction of fixed points of contractive type mappings in Banach space, C.I.M.E. Lecture Notes, Ispra, Italy, 1967.

29. - On the approximation solvability of nonlinear equations, Math. Ann. 177 (1968), 156-164.

30. N. I. Polsky, Projection methods in applied mathematics, Dokl. Akad. Nauk SSSR 143 (1962), 787-790=Soviet Math. Dokl. 3 (1962), 488.

31. F. Riesz and B. Sz.-Nagy, Functional analysis, Ungar, New York, 1955.

32. E. Rothe, Zur Theorie der topologischen Ordnung und der Vektorfelder in Banachschen Räumen, Compositio Math. 5 (1937), 177-197.

33. H. Schaefer, Uber die Methode sukzessiver Approximationen, Jber. Deutsch. Math.Verein. 59 (1957), 131-140.

34. J. Schauder, Der Fixpunktsatz in Functionalräumen, Studia Math. 2 (1930), 171-180.

35. A. E. Taylor, Introduction to functional analysis, Wiley, New York, 1958.

36. T. S. Tucker, Leray-Schauder theorem for P-compact operators and its consequences, J. Math. Anal. Appl. (to appear).

37. A. Tychonoff, Ein Fixpunktsatz, Math. Ann. 111 (1935), 767-776.

38. M. M. Vainberg, Variational methods for the study of nonlinear operators, Holden-Day, San Francisco, Calif., 1964.

39. G. Vainikko, On the convergence of collocation method for nonlinear differential equations, J. Comp. Math. Math. Phys. 6 (1966), 35-42.

40. S. Yamamuro, Some fixed point theorems in locally convex linear spaces, Yokohama Math. J. 11 (1963), 5-12.

41. E. H. Zarantonello, Solving functional equations by contractive averaging, Tech. Rep. No. 160, U.S. Army Res. Center, Madison, Wisconsin, 1960.

\section{University OF Chicago,} Chicago, IllinoIs 